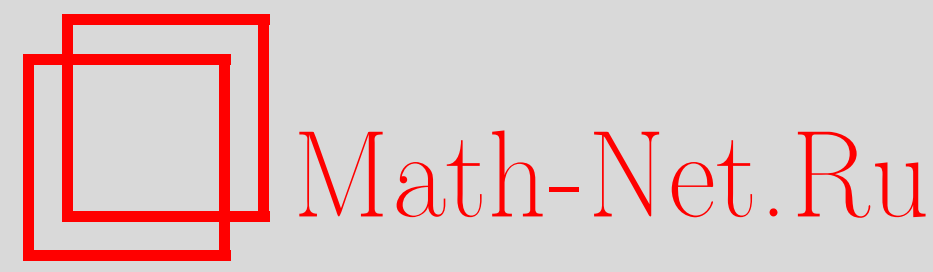

Ю. А. Неретин, Индексное гипергеометрическое преобразование и имитация анализа ядер Березина на гиперболических пространствах, Матем. сб., 2001, том 192, номер 3, 83-114

DOI: https://doi.org/10.4213/sm552

Использование Общероссийского математического портала Math-Net.Ru подразумевает, что вы прочитали и согласны с пользовательским соглашением

http://www. mathnet.ru/rus/agreement

Параметры загрузки:

IP : 54.198 .64 .247

26 апреля 2023 г., 16:08:31 
УДК 519.46

\author{
Ю. А. Неретин
}

\title{
Индексное гипергеометрическое преобразование и имитация анализа ядер Березина на гиперболических пространствах
}

\begin{abstract}
Индексное гипергеометрическое преобразование (известное также как преобразование Олевского или преобразование Якоби) обобщает сферическое преобразование в $L^{2}$ на симметрических пространствах ранга 1 (т.е. вещественных, комплексных и кватернионных пространствах Лобачевского). Цель настоящей статьи - получить свойства индексного гипергеометрического преобразования, имитирующие анализ ядер Березина на таких пространствах.

Обсуждается также задача о построении унитарного оператора, отождествляющего $L^{2}$ и пространство Березина. Задача приводит к некоторому интегральному выражению ( $\Lambda$-функции), которое, по-видимому, не выражается с помощью конечного числа операций через стандартные спецфункции (лишь при некоторых выделенных значениях параметра сводится к так называемым функциям типа Вольтерра). Исследуются свойства этого вьражения. Показьвается, что для некоторых серий симметрических пространств болшшого ранга этот же оператор унитарной эквивалентности записьвается через определители, составленнъе из $\Lambda$-функций.
\end{abstract}

Библиографоия: 41 название.

\section{Введение}

0.1. Индексное гипергеометрическое преобразование МелераГ. Вейля-Титчмарша-М.Н. Олевского. Фиксируем $b, c>0$. Рассмотрим интегральное преобразование $J_{b, c}$, заданное формулой

$$
\begin{aligned}
g(s) & =J_{b, c} f(s) \\
& =\frac{1}{\Gamma(b+c)} \int_{0}^{\infty} f(x)_{2} F_{1}(b+i s, b-i s ; b+c ;-x) x^{b+c-1}(1+x)^{b-c} d x,
\end{aligned}
$$

где ${ }_{2} F_{1}(\cdot)$ - гипергеометрическая функция Гаусса. Обратный оператор $J_{b, c}^{-1}$ задается формулой

$$
\begin{aligned}
f(x) & =J_{b, c}^{-1} g(x) \\
& =\frac{1}{\pi \Gamma(b+c)} \int_{0}^{\infty} g(s){ }_{2} F_{1}(b+i s, b-i s ; b+c ;-x)\left|\frac{\Gamma(b+i s) \Gamma(c+i s)}{\Gamma(2 i s)}\right|^{2} d s .
\end{aligned}
$$

Работа выполнена при поддержке Российского фонда фундаментальных исследований (грант № 98-01-00303) и NWO (грант № 047-008-009). 
Эта пара взаимно обратных преобразований была обнаружена Вейлем[1] в 1910 г. ${ }^{1}$ Результат Вейля не привлек особого внимания, и снова эти преобразования появляются у Титчмарша [3] в 1946 г. и М.Н. Олевского [4] в 1949 г. Основной причиной интереса к преобразованию (0.1) в последуюшие годы было то, что сферические преобразования для всех некомпактных римановых симметрических пространств ранга 1 имеют вид (0.1) при подходящих значениях параметров $b, c$.

Подробнее о преобразовании (0.1) см. статьи Коорнвиндера и Фленстед-Йенсена [5]-[8], а также книги Якубовича, Лучко [9], Самко, Килбаса, Маричева [10] и том 5 таблиц Прудникова, Брычкова, Маричева [11]; обзор Коорнвиндера [7] содержит обширную библиографию.

О различных обобшениях см., например, работы Якубовича, Лучко [9] (преобразование Уимпа с $G$-функцией), Кулинка, Стокмана [12] (q-аналоги), Хекмана, Опдама [13]-[15] (многомерные аналоги).

Для преобразования (0.1) в математической литературе используются термины

- индексное гипергеометрическое преобразование,

- обобщенное преобразование Фурье,

- преобразование Олевского,

- преобразование Якоби, ${ }^{2}$

- преобразование Фурье-Якоби.

0.2. Содержание работы. Пространство $L^{2}$ на некомпактном римановом симметрическом пространстве $G / K$ допускает естественную деформацию, обнаруженную (в случае эрмитовых симметрических пространств) Березиным в [16], см. работы [17]-[24] и библиографию в [23]. При малом значении параметра деформации (или при большом значении нашего параметра $\theta$, см. ниже п. 3.2) представления Березина группы $G$ эквивалентны представлению $G$ в $L^{2}(G / K)$.

Формула Планшереля для этой деформации в случае пространств ранга 1 была получена ван Дейком и Хилле в [19], а в общем случае - автором в [21]-[23].

Одной из целей работы было понять, как отзываются эти результаты на теории индексного гипергеометрического преобразования.

Второй целью было построение явного унитарного сплетающего оператора из пространства $L^{2}(G / K)$ в пространство Березина при большом значении параметра $\theta$. Мы пытаемся что-либо понять об этом операторе, используя индексное преобразование.

Параграф̆ 1 настоящей работы содержит предварительные сведения по индексному гипергеометрическому преобразованию.

В $\S 2$ мы вычисляем образы операторов

$$
f(x) \mapsto x f(x), \quad f(x) \mapsto x(x+1) \frac{d}{d x} f(x)
$$

при индексном преобразовании. Оказывается, что эти операторы переходят в разностные операторы мнимого направления. Например, оператору умножения на $x$

\footnotetext{
${ }^{1}$ Частный случай $b=1 / 2, c=1$ (преобразование Мелера-Фока) был обнаружен Мелером [2] в 1881 г.

2 Термин введен Коорнвиндером по ассоциации с разложением в рядпо многочленам Якоби.
} 
соответствует оператор

$$
P g(s)=\frac{(b-i s)(c-i s)}{(-2 i s)(1-2 i s)}(g(s+i)-g(s))+\frac{(b+i s)(c+i s)}{(2 i s)(1+2 i s)}(g(s-i)-g(s)) .
$$

Хотя эти результаты очень просты, нам не удалось найти в литературе утверждения об операторе $x(x+1) \frac{d}{d x} f(x)$. Первое утверждение является очень частным случаем одной недавней теоремы Чередника [25].

В $\S 3$ мы даем краткий обзор по анализу ядер Березина на симметрических пространствах ранга 1. Этот раздел в дальнейшем имитируется на уровне индексного гипергеометрического преобразования.

Параграф 4 содержит предварительные сведения о непрерывных двойственных многочленах Хана.

В $\S 5$ мы получаем серию нестандартных формул Планшереля. Точнее, мы показываем, что индексное преобразование является унитарным оператором из некоторого естественного пространства голоморфных функций в круге (или на полуплоскости) в пространство $L^{2}$ на полупрямой по некоторой мере. Этот параграф и является имитацией работ [19], [21] на уровне спецфункций одной переменной.

В $\S 6$ мы вводим функцию

$$
\begin{gathered}
\Lambda_{b, c}^{a}(x)=\frac{1}{\pi \Gamma(b+c)} \int_{0}^{\infty} \frac{\Gamma(a+i s) \Gamma(b+i s) \Gamma(b-i s) \Gamma(c+i s) \Gamma(c-i s)}{\Gamma(2 i s) \Gamma(-2 i s)} \\
\times{ }_{2} F_{1}(b+i s, b-i s ; b+c ;-x) d s .
\end{gathered}
$$

Целью является построение упомянутого выше унитарного оператора из $L^{2}$ в пространство Березина. По-видимому, функция $\Lambda$ не выражается явно через известные спецфункции (за исключением некоторых частных случаев, когда ответ выражается через редко употребляемые функции типа Вольтерра). Далее мы пытаемся дать ответ на неф̆ормальный вопрос о том, стоит ли считать $\Lambda$-функцию новой спецфункцией. Мы выводим ряд довольно забавных тождеств с $\Lambda$-функцией, а в $\S 7$ строим с помощью $\Lambda$-функции ортогональные базисы в $L^{2}$ на полупрямой.

В $\S 8$ мы без доказательства приводим решение задачи об явной унитарной эквивалентности между $L^{2}$ и деформацией Березина в случае симметрических пространств

$$
\mathrm{U}(p, q) /(\mathrm{U}(p) \times \mathrm{U}(q)) .
$$

Оказывается, что ответ выписывается через определитель, составленньй из $\Lambda$ функций. Этот параграф объясняет цели введения $\Lambda$-функции.

0.3. Обозначения: $\mathbb{R}_{+}-$полупрямая $x \geqslant 0$;

$$
{ }_{2} F_{1}(a, b ; c ; x):=\sum_{n=0}^{\infty} \frac{(a)_{n}(b)_{n}}{(c)_{n} n !} x^{n}
$$

- гипергеометрическая функция Гаусса;

$$
{ }_{p} F_{q}\left[\begin{array}{l}
a_{1}, \ldots, a_{p} \\
c_{1}, \ldots, c_{q}
\end{array} ; x\right]:=\sum_{n=0}^{\infty} \frac{\left(a_{1}\right)_{n} \cdots\left(a_{p}\right)_{n}}{\left(c_{1}\right)_{n} \cdots\left(c_{q}\right)_{n} n !} x^{n}
$$

- обобщенная гипергеометрическая функция. 


\section{§1. Предварительные сведения}

1.1. Определение. Фиксируем $b, c>0$. Пусть $f(x)$ - функция на полупрямой. Ее индексное гипергеометрическое преобразование $J_{b, c} f$ (мы также используем обозначение $[\widehat{f}]_{b, c}$ ) задается формулой

$$
\begin{aligned}
g(s) & =J_{b, c} f(s)=[\widehat{f}(s)]_{b, c} \\
& =\frac{1}{\Gamma(b+c)} \int_{0}^{\infty} f(x)_{2} F_{1}(b+i s, b-i s ; b+c ;-x) x^{b+c-1}(1+x)^{b-c} d x .
\end{aligned}
$$

Формула обращения для индексного гипергеометрического преобразования имеет вид

$$
\begin{aligned}
f(x) & =J_{b, c}^{-1} g(x) \\
& =\frac{1}{\pi \Gamma(b+c)} \int_{0}^{\infty} g(s){ }_{2} F_{1}(b+i s, b-i s ; b+c ;-x)\left|\frac{\Gamma(b+i s) \Gamma(c+i s)}{\Gamma(2 i s)}\right|^{2} d s .
\end{aligned}
$$

Равносильное утверждение: оператор $J_{b, c}$ является унитарным оператором

$$
L^{2}\left(\mathbb{R}_{+}, x^{b+c-1}(1+x)^{b-c} d x\right) \rightarrow L^{2}\left(\mathbb{R}_{+},\left|\frac{\Gamma(b+i s) \Gamma(c+i s)}{\Gamma(2 i s)}\right|^{2} d x\right) .
$$

Запишем также условие унитарности (формула Планшереля) в явном виде:

$$
\begin{aligned}
\int_{0}^{\infty} & f_{1}(x) \overline{f_{2}(x)} x^{b+c-1}(1+x)^{b-c} d x \\
\quad= & \frac{1}{\pi} \int_{0}^{\infty}\left[\widehat{f}_{1}(s)\right]_{b, c}{\overline{\left[\hat{f}_{2}(s)\right.}}_{b, c}\left|\frac{\Gamma(b+i s) \Gamma(c+i s)}{\Gamma(2 i s)}\right|^{2} d s .
\end{aligned}
$$

Свойства оператора $J_{b, c}$ в различных функциональных пространствах подробно изучались, см. обзор [7], а также [9].

ЗАмечАниЕ. Предложенный Вейлем [1] вывод формулы обрашения (1.2) довольно прост. Рассмотрим гипергеометрический дифференциальный оператор

$$
D:=x(x+1) \frac{d^{2}}{d x^{2}}+[(c+b)+(2 b+1) x] \frac{d}{d x}+b^{2}
$$

на $\mathbb{R}_{+}$. Функции ${ }_{2} F_{1}(b+i s, b-i s ; b+c ;-x)$ - это в точности собственные функции оператора $D$. Для оператора $D$ может быть явно написана резольвента (она же формула Грина) $R(\lambda)=(D-\lambda)^{-1}$ (см. работы Миллер-Лебедевой [26], [27]). Зная резольвенту, несложно уже получить спектральное разложение оператора по формуле

$$
D=\frac{1}{2 \pi i} \int \lim _{\varepsilon \rightarrow 0}[R(\lambda+i \varepsilon)-R(\lambda-i \varepsilon)] d \lambda .
$$

Подробное обсуждение этого обшего приема см. у Данфорда, Шварца [28]. В приложении к дифференциальным операторам (и, в частности, к гипергеометрическому оператору) этот метод излагается у Титчмарша [3].

О других выводах формулы обрашения см. [4], [6]-[10]. 
ЗАмечАниЕ. Очевидно, верно равенство

$$
\widehat{[D f]}=s^{2}[\widehat{f}(s)]
$$

\section{2. Голоморфное продолжение в полосу.}

ЛЕмма 1.1. Пусть $f$ интегрируема на $\mathbb{R}_{+} u$

$$
f(x)=o\left(x^{-\alpha-\varepsilon}\right), \quad x \rightarrow+\infty
$$

где $\varepsilon>0$. Тогда $[\widehat{f}(s)]_{b, c}$ голоморфна в полосе

$$
|\operatorname{Im} s|<\alpha-b
$$

ДокАЗАтЕльство. Утверждение вытекает из следующей асимптотики для гипергеометрической функции (см. [29; том $1,(2.3 .2 .9)])$ при $x \rightarrow+\infty$ :

${ }_{2} F_{1}(b+i s, b-i s ; b+c ;-x)=\lambda_{1} x^{-b+i s}+\lambda_{2} x^{-b-i s}+O\left(x^{-b+i s-1}\right)+O\left(x^{-b-i s-1}\right)$,

где $2 s \notin \mathbb{Z}$, а $\lambda_{1}, \lambda_{2}$ - некоторые константы (при $2 s \in \mathbb{Z}$ появляется дополнительно множитель $\ln x$ при старшем слагаемом).

ЗАмечАнИЕ. Достаточным условием вхождения непрерывной функции $f(x)$ в $L^{2}$ по мере $x^{b+c-1}(1+x)^{b-c} d x$ является убывание

$$
f(x)=o\left(x^{-b-\varepsilon}\right), \quad x \rightarrow+\infty
$$

и мы видим, что это условие обеспечивает существование аналитического продолжения в достаточно узкую полосу.

1.3. Преобразование Фурье. Для некоторых значений параметров $b, c$ ядро ${ }_{2} F_{1}(b+i s, b-i s ; b+c ;-x)$ является элементарной функцией. В частности (см. $[29$; том $1,(2.8 .11),(2.8 .12)])$,

$$
\begin{gathered}
{ }_{2} F_{1}\left(i s,-i s ; \frac{1}{2} ;-x\right)=\cos (2 s \operatorname{arcsh} \sqrt{x}), \\
{ }_{2} F_{1}\left(\frac{1}{2}+i s, \frac{1}{2}-i s ; \frac{3}{2} ;-x\right)=\frac{\sin (2 s \operatorname{arcsh} \sqrt{x})}{2 s \sqrt{x}} .
\end{gathered}
$$

Поэтому

$$
\begin{aligned}
& J_{0,1 / 2} f(s)=\frac{1}{\Gamma(1 / 2)} \int_{0}^{\infty} f(x) \cos (2 s \operatorname{arcsh} \sqrt{x}) x^{-1 / 2}(1+x)^{-1 / 2} d x \\
& J_{1 / 2,1} f(x)=\frac{1}{\Gamma(3 / 2)} \int_{0}^{\infty} f(x) \frac{\sin (2 s \operatorname{arcsh} \sqrt{x})}{2 s \sqrt{x}} x^{1 / 2}(1+x)^{-1 / 2} d x .
\end{aligned}
$$


Замена переменной $x=\operatorname{sh}^{2} y$ приводит эти интегралы к виду

$$
\begin{aligned}
& J_{0,1 / 2} f(s)=\frac{2}{\Gamma(1 / 2)} \int_{0}^{\infty} f\left(\operatorname{sh}^{2} y\right) \cos (2 s y) d y \\
& J_{1 / 2,1} f(s)=\frac{1}{\Gamma(3 / 2)} \int_{0}^{\infty} f\left(\operatorname{sh}^{2} y\right) \frac{\operatorname{sh} y \sin (2 s y)}{2 s} d y
\end{aligned}
$$

т.е. операторы $J_{0,1 / 2}, J_{1 / 2,1}$ отличаются соответственно от синус-преобразования Фрье и косинус-преобразования Фурье несушественной заменой переменной.

Отметим, что элементарными являются и все функции вида

$$
{ }_{2} F_{1}\left(k+i s, k-i s ; \frac{1}{2}+k+l ;-x\right),{ }_{2} F_{1}\left(\frac{1}{2}+k+i s, \frac{1}{2}+k-i s ; \frac{3}{2}+k+l ;-x\right)
$$

при целых $k, l$. Они получаются из $(1.3),(1.4)$ применением подходящих явно выписываемых дифференциальных операторов (см. [29; том 1, 2.8, формулы $(20),(22)$, $(24),(27)])$.

1.4. Сферические преобразования. Через $\mathbb{K}$ мы обозначим $\mathbb{R}, \mathbb{C}$ или тело кватернионов $\mathbb{H}$. Через $r$ мы обозначим размерность тела $\mathbb{K}$, через $\mathbb{K}^{n}-n$-мерное пространство над $\mathbb{K}$ со стандартным скалярным произведением:

$$
\langle z, u\rangle=\sum z_{j} \bar{u}_{j}
$$

Через $\mathrm{U}(1, n ; \mathbb{K})$ мы обозначим псевдоунитарную группу над $\mathbb{K}$, т.е. группу $(1+n) \times(1+n)$-матриц $\left(\begin{array}{ll}a & b \\ c & d\end{array}\right)$ над $\mathbb{K}$, удовлетворяющих условию

$$
\left(\begin{array}{ll}
a & b \\
c & d
\end{array}\right)\left(\begin{array}{cc}
-1 & 0 \\
0 & 1
\end{array}\right)\left(\begin{array}{ll}
a & b \\
c & d
\end{array}\right)^{*}=\left(\begin{array}{cc}
-1 & 0 \\
0 & 1
\end{array}\right)
$$

Стандартные обозначения для групा $\mathrm{U}(1, n ; \mathbb{K})$ в случаях $\mathbb{K}=\mathbb{R}, \mathbb{C}, \mathbb{H}$ соответственно: $\mathrm{O}(1, n), \mathrm{U}(1, n), \mathrm{Sp}(1, n)$.

Через $\mathrm{B}_{n}(\mathbb{K})$ мы обозначим шар $\langle z, z\rangle<1$ в $\mathbb{K}^{n}$. Через $S^{r n-1}$ мы обозначим сфферу $\langle z, z\rangle=1$. Группа $\mathrm{U}(1, n ; \mathbb{K})$ действует на $\mathrm{B}_{n}(\mathbb{K})$ дробно-линейными преобразованиями

$$
z \mapsto z^{[g]}:=(a+z c)^{-1}(b+z d) .
$$

Стабилизатор $K$ точки $0 \in \mathrm{B}_{n}(\mathbb{K})$ состоит из матриц вида

$$
\left(\begin{array}{ll}
a & 0 \\
0 & d
\end{array}\right), \quad|a|=1, \quad d \in \mathrm{U}(n ; \mathbb{K}) .
$$

Поэтому $\mathrm{B}_{n}(\mathbb{K})$ есть однородное пространство:

$$
\mathrm{B}_{n}(\mathbb{K})=\mathrm{U}(1, n ; \mathbb{K}) /(\mathrm{U}(1 ; \mathbb{K}) \times \mathrm{U}(n ; \mathbb{K})) \text {. }
$$

Якобиан преобразования (1.5) равен

$$
J(g ; z)=|a+z c|^{-r(1+n)} .
$$


Отметим простую формулу

$$
1-\left\langle z^{[g]}, u^{[g]}\right\rangle=(a+z c)^{-1}\left(1-\langle z, u\rangle \overline{(a+u c)}^{-1} .\right.
$$

Отсюда видно, что $\mathrm{U}(1, n ; \mathbb{K})$-инвариантная мера на $\mathrm{B}_{n}(\mathbb{K})$ имеет вид

$$
d m(z)=(1-\langle z, z\rangle)^{-(n+1) r / 2} d z
$$

где через $d z$ обозначена мера Лебега на $\mathrm{B}_{n}(\mathbb{K})$.

Группа $\mathrm{U}(1, n ; \mathbb{K})$ действует в $L^{2}\left(\mathrm{~B}_{n}(\mathbb{K}), d m(z)\right)$ заменами переменной

$$
f(z) \mapsto f\left((a+z c)^{-1}(b+z d)\right)
$$

Рассмотрим задачу о разложении $L^{2}\left(\mathrm{~B}_{n}(\mathbb{K}), d m(z)\right)$ в прямой интеграл неприводимых представлений $\mathrm{U}(1, n ; \mathbb{K})$.

Пусть $s \in \mathbb{R}$. Представление $T_{s}$ основной унитарной серии групшы $\mathrm{U}(1, n ; \mathbb{K})$ реализуется в $L^{2}\left(S^{r n-1}\right)$ и задается формулой

$$
T_{s}\left(\begin{array}{ll}
a & b \\
c & d
\end{array}\right) f(h)=f\left((a+h c)^{-1}(b+h d)\right)|a+h c|^{-(n+1) r / 2+1+i s}
$$

где $h \in S^{r n-1}$.

Рассмотрим оператор $A$ из пространства $L^{2}\left(\mathrm{~B}_{n}(\mathbb{K}), d m(z)\right)$ в пространство функций на $S^{r n-1} \times \mathbb{R}_{+}$, заданньй формулой

$$
A f(h, s)=\int_{\mathrm{B}_{n}(\mathbb{K})} f(z) \frac{|1-\langle z, h\rangle|^{-(n+1) r / 2+1+i s}}{|1-\langle z, z\rangle|^{(n+1) r / 4+1 / 2+i s / 2}} d z .
$$

Если $f$ преобразуется по формуле $(1.7)$, то $G(h, s):=A f(h, s)$ преобразуется как

$$
G(h, s) \mapsto G\left((a+h c)^{-1}(b+h d), s\right)|a+h c|^{-(n+1) r / 2+1+i s} .
$$

Теперь мы хотим построить меру $d \nu$ (она называется мерой Планшереля) на $S^{r n-1} \times \mathbb{R}_{+}$такую, что оператор $A$ является унитарным оператором

$$
L^{2}\left(\mathrm{~B}_{n}(\mathbb{K}), d m(z)\right) \rightarrow L^{2}\left(S^{r n-1} \times \mathbb{R}_{+}, d \nu\right)
$$

(это и решит задачу разложения в прямой интеграл). Очевидно, множитель $|a+h c|$ (см. (1.8), (1.9)) равен единице, если матрица $\left(\begin{array}{ll}a & b \\ c & d\end{array}\right)$ имеет вид (1.6). Следовательно, искомая мера $d \nu$ инвариантна относительно группы $\mathrm{U}(n ; \mathbb{K})$, а поэтому плотность искомой меры имеет вид

$$
\sigma(s) d s d h
$$

Чтобы найти функцию $\sigma(s)$, мы рассмотрим всевозможные функции $f$ на $\mathrm{B}_{n}(\mathbb{K})$, зависяшие лишш от радиуса $|z|$. Удобно ввести переменную

$$
x=\frac{|z|^{2}}{1-|z|^{2}}
$$


и положить $f=f(x)$. Тогда соответствующая функция $G(h, s)$ зависит лишш от переменной $s$, и несложное вычисление дает формулу

$$
G(s)=\text { const } \cdot \int_{0}^{\infty} f(x)_{2} F_{1}(b+i s, b-i s ; b+c ;-x) x^{b+c-1}(1+x)^{b-c} d x
$$

где

$$
b=\frac{(n+1) r}{4}-\frac{1}{2}, \quad c=\frac{(n-1) r}{4}+\frac{1}{2} .
$$

Это интегральное преобразование, определенное на $\mathrm{U}(n ; \mathbb{K})$-инвариантных функциях на $\mathrm{B}_{n}(\mathbb{K})$, называется сферическим преобразованием. Мы видим, что сферические преобразования являются частными случаями индексного гипергеометрического преобразования. Теперь формула обращения (1.2) дает следующую плотность меры Планшереля:

$$
\sigma(s)=\left|\frac{\Gamma(b+i s) \Gamma(c+i s)}{\Gamma(2 i s)}\right|^{2}
$$

\section{§ 2. Соответствие некоторых операторов}

\section{1. Оператор умножения на $x$.}

ТЕОРема 2.1. Пусть функция $f$ на $\mathbb{R}^{+}$непрерывна и удовлетворяет условию

$$
f(x)=o\left(x^{-b-1-\varepsilon}\right), \quad x \rightarrow+\infty .
$$

Тогда

$$
[\widehat{x f(x)}]_{b, c}=P[\widehat{f(x)}]_{b, c},
$$

әде разностный оператор Pg задан формулой

$$
P g(s)=\frac{(b-i s)(c-i s)}{(-2 i s)(1-2 i s)}(g(s+i)-g(s))+\frac{(b+i s)(c+i s)}{(2 i s)(1+2 i s)}(g(s-i)-g(s)) \text {. }
$$

ДокАЗАТЕльство. Условие (2.1) обеспечивает существование голоморфного продолжения функции $\widehat{f}$ в полосу

$$
\operatorname{Im} s<1+\varepsilon
$$

Само равенство (2.2) равносильно следующему тождеству для гипергеометрических функций

$$
\begin{aligned}
x_{2} F_{1}(b+i s, b-i s ; b+c ;-x) \\
=\frac{(b-i s)(c-i s)}{(-2 i s)(1-2 i s)}{ }_{2} F_{1}(b-1+i s, b+1-i s ; b+c ;-x) \\
\quad-\left[\frac{(b-i s)(c-i s)}{(-2 i s)(1-2 i s)}+\frac{(b+i s)(c+i s)}{(2 i s)(1+2 i s)}\right]{ }_{2} F_{1}(b+i s, b-i s ; b+c ;-x) \\
\quad+\frac{(b+i s)(c+i s)}{(2 i s)(1+2 i s)}{ }_{2} F_{1}(b+1+i s, b-1-i s ; b+c ;-x),
\end{aligned}
$$


которое, в свою очередь, равносильно следующему соотношению между ассоциированными гипергеометрическими функциями ${ }^{3}$ :

$$
\begin{aligned}
-y_{2} F_{1}(p, q ; r ; y) \\
=\frac{q(r-p)}{(q-p)(1+q-p)}{ }_{2} F_{1}(p-1, q+1 ; r ; y) \\
\quad-\left[\frac{q(r-p)}{(q-p)(1+q-p)}+\frac{p(r-q)}{(p-q)(1+p-q)}\right]{ }_{2} F_{1}(p, q ; r ; y) \\
\quad+\frac{p(r-q)}{(p-q)(1+p-q)}{ }_{2} F_{1}(p+1, q-1 ; r ; y) .
\end{aligned}
$$

Последнее равенство отсутствует в стандартных таблицах, но легко проверяется приравниванием коэффициентов тейлоровского разложения по $y$. Действительно,

$$
\begin{aligned}
{ }_{2} F_{1} & (p-1, q+1 ; r ; y)-{ }_{2} F_{1}(p, q ; r ; y) \\
& =\sum\left(\frac{(p-1)_{k}(q+1)_{k}}{(r)_{k} k !}-\frac{(p)_{k}(q)_{k}}{(r)_{k} k !}\right) y_{k} \\
& =\sum \frac{(p)_{k}(q)_{k}}{(r)_{k} k !}\left(\frac{(p-1)(q+k)}{(p+k-1) q}-1\right) y_{k} \\
& =\sum \frac{(p)_{k}(q)_{k}}{(r)_{k} k !} \cdot \frac{-k(1+q-p)}{q(p+k-1)} y_{k} .
\end{aligned}
$$

Преобразуя таким же образом ${ }_{2} F_{1}(p+1, q-1 ; r ; y)-{ }_{2} F_{1}(p, q ; r ; y)$, мы приводим правую часть $(2.3)$ к виду

$$
\sum \frac{(p)_{k}(q)_{k}}{(r)_{k} k !}\left(\frac{-k(r-p)}{(q-p)(p+k-1)}-\frac{-k(r-q)}{(p-q)(q+k-1)}\right) y_{k}
$$

а последнее уже равно левой части (2.3).

\section{2. Дифференцирование.}

ТЕОРЕМА 2.2. Пусть $f$ и $f^{\prime}$ непрерывны и удовлетворяют условиям убивания (2.2). Тогда

$$
\left[x\left(\widehat{x+1)} \frac{d}{d x} f\right]_{b, c}=H[\widehat{f}]_{b, c},\right.
$$

где разностный оператор $Н$ задан формулой

$$
\begin{aligned}
H g(s)= & \frac{(b-i s)(b+1-i s)(c-i s)}{(-2 i s)(1-2 i s)}(g(s+i)-g(s)) \\
& +\frac{(b+i s)(b+1+i s)(c+i s)}{(+2 i s)(1+2 i s)}(g(s-i)-g(s))-(b+c) g(s) .
\end{aligned}
$$

\footnotetext{
${ }^{3}$ Для любых трех гипергеометрических функций ${ }_{2} F_{1}(p, q ; r ; y),{ }_{2} F_{1}\left(p+k_{1}, q+l_{1}\right.$; $\left.r+m_{1} ; y\right),{ }_{2} F_{1}\left(p+k_{2}, q+l_{2} ; r+m_{2} ; y\right)$ с цельми $k_{j}, l_{j}, m_{j}$ существует линейная зависимость с полиномиальными по $y$ коэффициентами.
} 
ДоКАЗАТЕЛЬСТво.

$$
\begin{aligned}
\int_{0}^{\infty} & \left\{x(x+1) \frac{d}{d x} f(x)\right\}{ }_{2} F_{1}(b+i s, b-i s ; b+c ;-x) x^{b+c-1}(1+x)^{b-c} d x \\
= & -\int_{0}^{\infty} f(x) \frac{d}{d x}\left\{x^{b+c}(1+x)^{b-c+1}{ }_{2} F_{1}(b+i s, b-i s ; b+c ;-x)\right\} d x \\
= & -\int_{0}^{\infty} f(x)\left\{x(x+1) \frac{d}{d x}{ }_{2} F_{1}(b+i s, b-i s ; b+c ;-x)\right. \\
& \left.+((2 b+1) x+(b+c)){ }_{2} F_{1}(b+i s, b-i s ; b+c ;-x)\right\} x^{b+c-1}(1+x)^{b-c} d x .
\end{aligned}
$$

Теперь все сводится к проверке тождества

$$
\begin{aligned}
& -\left\{x(x+1) \frac{d}{d x}+(2 b+1) x+(b+c)\right\}{ }_{2} F_{1}(b+i s, b-i s ; b+c ;-x) \\
& =\frac{(b-i s)(b+1-i s)(c-i s)}{(-2 i s)(1-2 i s)}{ }_{2} F_{1}(b-1+i s, b+1-i s ; b+c ;-x) \\
& -\left(\frac{(b-i s)(b+1-i s)(c-i s)}{(-2 i s)(1-2 i s)}+\frac{(b+i s)(b+1+i s)(c+i s)}{(+2 i s)(1+2 i s)}-b-c\right) \\
& \quad \times{ }_{2} F_{1}(b+i s, b-i s ; b+c ;-x) \\
& +\frac{(b+i s)(b+1+i s)(c+i s)}{(+2 i s)(1+2 i s)}{ }_{2} F_{1}(b+1+i s, b-1-i s ; b+c ;-x) .
\end{aligned}
$$

Оно, в свою очередь, является соотношением между ассоциированньми функциями

$$
\begin{aligned}
& -\left\{x(x+1) \frac{d}{d x}+(p+q+1) x+r\right\}{ }_{2} F_{1}(p, q ; r ;-x) \\
& =\left(x^{2}+x\right) \frac{p q}{r}{ }_{2} F_{1}(p+1, q+1 ; r+1 ;-x)-(p+q+1) x_{2} F_{1}(p, q ; r ;-x) \\
& =\frac{q(q+1)(r-p)}{(1+q-p)(p-q)}\left({ }_{2} F_{1}(p-1, q+1 ; r ;-x)-{ }_{2} F_{1}(p, q ; r ;-x)\right) \\
& \quad+\frac{p(p+1)(r-q)}{(1+p-q)(q-p)}\left({ }_{2} F_{1}(p+1, q-1 ; r ;-x)-{ }_{2} F_{1}(p, q ; r ;-x)\right),
\end{aligned}
$$

которое довольно легко проверяется приравниванием тейлоровских коэффициентов при $x^{k}$.

\section{§3. Ядра Березина}

Пространство $L^{2}$ на шаре $\mathrm{B}_{n}(\mathbb{K})$, т.е. на симметрическом пространстве

$$
\mathrm{U}(1, n ; \mathbb{K}) /(\mathrm{U}(1, \mathbb{K}) \times \mathrm{U}(n, \mathbb{K})),
$$

допускает естественную деформацию, которая сейчас будет описана (подробнее см. [19] для симметрических пространств ранга 1 и [23] для симметрических пространств произвольного ранга). Основная цель настоящей работы состоит в имитации этой деформации на уровне спецфункций. 
3.1. Положительно определенные ядра. Напомним определение положительно определенного ядра (подробное обсуждение и ссылки см. в [23]). Пусть $X-$ множество. Функция $L(x, y)$ на $X \times X$ называется положстельно определенным ядром, если $L(y, x)=\overline{L(x, y)}$ и для любых $x_{1}, \ldots, x_{k} \in X$ матрица

$$
\operatorname{det}\left(\begin{array}{ccc}
L\left(x_{1}, x_{1}\right) & \ldots & L\left(x_{1}, x_{k}\right) \\
\vdots & \ddots & \vdots \\
L\left(x_{k}, x_{1}\right) & \ldots & L\left(x_{k}, x_{k}\right)
\end{array}\right)
$$

неотрицательно определена.

Пусть $L$ - положительно определенное ядро на множестве $X$. Тогда сушествует гильбертово пространство $H=H[L]$ и система векторов $v_{x} \in H$, нумеруемая точками $x \in X$, такая, что

1) линейная оболочка векторов $v_{x}$ плотна в $H[L]$,

2) $\left\langle v_{x}, v_{y}\right\rangle=L(y, x)$.

Далее, каждому вектору $h \in H[L]$ мы поставим в соответствие функцию $f_{h}(x)$ на $X$ по формуле

$$
f_{h}(x)=\left\langle h, v_{x}\right\rangle
$$

Таким образом, пространство $H$ реализуется как некоторое пространство функций на $X$, это пространство функций мы обозначим через $H^{\circ}[L]$. Очевидно, векторам $v_{a} \in H[L]$ соответствуют функции

$$
\varphi_{a}(x)=L(x, a)
$$

при этом для любой $f \in H^{\circ}[L]$ выполнено воспроизводящее свойство

$$
\left\langle f, \varphi_{a}\right\rangle_{H^{\circ}[L]}=f(a) .
$$

Это тождество дает некоторое, не вполне конструктивное, описание скалярного произведения в $H^{\circ}[L]$. Говорят также, что $L-$ воспроизводящее ядро пространства $H^{\circ}[L]$.

3.2. Ядра Березина. Ядро Березина $L_{\theta}(z, u)$ на $\mathrm{B}_{n}(\mathbb{K})$ определяется формулой

$$
L_{\theta}(z, u)=|1-\langle z, u\rangle|^{-\theta} .
$$

В случае $\mathbb{K}=\mathbb{R}, \mathbb{C}$ ядро $L_{\theta}(z, u)$ положительно определено для всех $\theta \geqslant 0$, а в случае $\mathbb{K}=\mathbb{H}-$ при $\theta \geqslant 2$. Группа $\mathrm{U}(1, n ; \mathbb{K})$ действует в $H^{\circ}\left(L_{\theta}\right)$ унитарными операторами по формуле

$$
T_{\theta}\left(\begin{array}{ll}
a & b \\
c & d
\end{array}\right) f(z)=f\left((a+z c)^{-1}(b+z d)\right)|a+z c|^{-\theta}
$$


3.3. Другой вариант определения. Рассмотрим ядро на $\mathrm{B}_{n}(\mathbb{K})$, заданное формулой

$$
M_{\theta}(z, u)=\frac{(1-\langle z, z\rangle)^{\theta / 2}(1-\langle u, u\rangle)^{\theta / 2}}{|1-\langle z, u\rangle|^{\theta}} .
$$

Рассмотрим связанные с этим ядром пространства $H\left(M_{\theta}\right), H^{\circ}\left(M_{\theta}\right)$. Группа $\mathrm{U}(1, n ; \mathbb{K})$ действует в пространстве $H^{\circ}\left[M_{\theta}\right]$ заменами переменной по формуле

$$
R_{\theta}\left(\begin{array}{ll}
a & b \\
c & d
\end{array}\right) f(z)=f\left((a+z c)^{-1}(b+z d)\right)
$$

Легко видеть, что конструкции пп. 3.2 и 3.3 равносильны. Канонический унитарный $\mathrm{U}(1, n ; \mathbb{K})$-сплетаюший оператор $H^{\circ}\left[L_{\theta}\right] \rightarrow H^{\circ}\left[M_{\theta}\right]$ - это просто оператор умножения на функцию $(1-\langle z, z\rangle)^{\theta / 2}$.

3.4. Более материальное описание пространства $H^{\circ}\left(L_{\theta}\right)$. Большой разницы между случаями $\mathbb{K}=\mathbb{R}, \mathbb{C}, \mathbb{H}$ нет, мы качестве основного примера будем рассматривать $\mathbb{K}=\mathbb{R}$. Дадим более конструктивное описание пространства $H^{\circ}\left(L_{\theta}\right)$ в этом случае (с дополнительным ограничением $\theta>n$ ).

Для этого рассмотрим пространство $V_{\theta}$ голоморфных функций в шаре $\mathrm{B}_{n}(\mathbb{C})$ со скалярным произведением

$$
\langle f, g\rangle_{V_{\theta}}=\frac{\Gamma(\theta)}{\pi^{n} \Gamma(\theta-n)} \int_{\mathrm{B}_{n}(\mathbb{C})} f(z) \overline{g(z)}(1-\langle z, z\rangle)^{\theta-n-1} d z .
$$

Универсальная накрываюшая группа группы $\mathrm{U}(1, n)=\mathrm{U}(1, n ; \mathbb{C})$ действует в пространстве $V_{\theta}$ унитарньми операторами по формуле

$$
\mathscr{U}_{\theta}\left(\begin{array}{ll}
a & b \\
c & d
\end{array}\right) f(z)=f\left((a+z c)^{-1}(b+z d)\right)(a+z c)^{-\theta}
$$

ЗАмечАниЕ. Для целого $\theta$ семейство операторов $\mathscr{U}_{\theta}$ задает линейное представление группы $\mathrm{U}(1, n)$. Если $\theta$ не является целым числом, то

$$
(a+z c)^{-\theta}=\left(1+z c a^{-1}\right)^{-\theta} e^{-\theta(\ln a+2 \pi k i)} .
$$

Легко показать, что $\left\langle c a^{-1}, c a^{-1}\right\rangle<1$, значит, $\left(1+z c a^{-1}\right)^{-\theta}$ канонически определено при $\langle z, z\rangle<1$. Поэтому оператор $\mathscr{U}_{\theta}\left(\begin{array}{ll}a & b \\ c & d\end{array}\right)$ определен с точностью до множителя $e^{2 \pi k i \theta}$, и в случае нецелого $\theta$ мы получаем проективное представление групшы $\mathrm{U}(1, n)$ или, что то же самое, линейное представление ее универсальной накрьваюшей.

Несложное вычисление (с интегралом Дирихле, см., например, [30; 1.8$])$ показывает, что функции $z_{1}^{k_{1}} \cdots z_{n}^{k_{n}}$ образуют ортогональный базис и

$$
\left\|z_{1}^{k_{1}} \cdots z_{n}^{k_{n}}\right\|^{2}=\frac{k_{1} ! \cdots k_{n} !}{(\theta)_{k_{1}+\cdots+k_{n}}}
$$


Рассмотрим функцию

$$
\varphi_{a}(z):=\left(1-\sum z_{j} \bar{a}_{j}\right)^{-\theta}=\sum_{k_{1}, \ldots, k_{n}} \frac{(\theta)_{k_{1}+\cdots+k_{n}} \bar{a}_{1}^{k_{1}} \cdots \bar{a}_{n}^{k_{n}}}{k_{1} ! \cdots k_{n} !} z_{1}^{k_{1}} \cdots z_{n}^{k_{n}} .
$$

Из равенств (3.4), (3.5) вытекает, что для любой функции $f(z)$, голоморфной в шаре, вьполнено

$$
\left\langle f, \varphi_{a}\right\rangle_{V_{\theta}}=f(a) .
$$

Итак, пространство $V_{\theta}$ определяется воспроизводящим ядром

$$
K(z, u)=(1-\langle z, u\rangle)^{-\theta} .
$$

Далее мы замечаем, что функция, голоморфная в $\mathrm{B}_{n}(\mathbb{C})$, однозначно определяется своим ограничением на $\mathrm{B}_{n}(\mathbb{R})$, поэтому мы можем рассматривать пространство $V_{\theta}$ как некоторое пространство функций на $\mathrm{B}_{n}(\mathbb{R})$. Это пространство функций как раз и определяется ядром Березина $L_{\theta}$ на $\mathrm{B}_{n}(\mathbb{R})$.

Еще раз подчеркнем, что в пространстве $H^{\circ}\left[L_{\theta}\right]$ на $\mathrm{B}_{n}(\mathbb{R})$ действует группа $\mathrm{U}(1, n)$, бо́льшая, чем $\mathrm{O}(1, n)^{4}$, подробнее см. [23].

3.5. Формула Планшереля. Отметим, что группа $\mathrm{U}(1, n ; \mathbb{K})$ действует в пространствах $L^{2}\left(\mathrm{~B}_{n}(\mathbb{K})\right)$ и во всех пространствах $H^{\circ}\left(M_{\theta}\right)$ по одной и той же формуле (1.7), (3.3). Рассмотрим следующий оператор, с точностью до функционального множителя совпадающий с оператором (1.9):

$$
\begin{aligned}
R_{\theta} f(h, s)= & \frac{1}{\Gamma(b+c)|\Gamma(\theta-b+i s)|^{2}} \\
& \times \int_{\mathrm{B}_{n}(\mathbb{K})} f(z) \frac{|1-\langle z, h\rangle|^{-(n+1) r / 2+1+i s}}{|1-\langle z, z\rangle|^{(n+1) r / 4+1 / 2+i s / 2}} d z .
\end{aligned}
$$

Тог да, как показано у ван Дейка и Хилле [19], при $\theta>b=(n+1) r / 4-1 / 2$ оператор $R_{\theta}$ является унитарньм оператором

$$
H^{\circ}\left(M_{\theta}\right) \rightarrow L^{2}\left(S^{r n-1} \times \mathbb{R}_{+}, \tau(s) d h d s\right),
$$

где $d h$ - мера Лебега на сфере, а

$$
\tau(s)=\frac{1}{\Gamma(\theta) \Gamma(\theta-b+c)}\left|\frac{\Gamma(\theta-b+i s) \Gamma(b+i s) \Gamma(c+i s)}{\Gamma(2 i s)}\right|^{2} .
$$

ЗАмЕчАниЕ. Если мы уберем выражение $\left|\Gamma^{2}(\theta-b+i s)\right|^{2}$ из знаменателя в (3.6), то в (3.7) это же выражение переместится в знаменатель; нормировка (3.6) по ряду причин более удобна.

В $§ 5$ мы получим теорему 5.3, имитируюшую формулу Планшереля (3.7) на уровне индексного преобразования; теорема ван Дейка и Хилле является частным случаем теоремы 5.3 .

\footnotetext{
${ }^{4} \mathrm{~B}$ случае комплексного шара аналогичной надгруппой является $\mathrm{U}(1, n) \times \mathrm{U}(1, n)$, а в случае кватернионного - соответственно $\mathrm{U}(2,2 n)$.
} 
3.6. Радиальные части пространств Березина. Как и в п. 3.4 , мы для определенности остановимся на случае $\mathbb{K}=\mathbb{R}$. Рассмотрим пространство голоморфных функций $V_{\theta}$, определенное в п. 3.4 , и в нем подпространство $V_{\theta}^{\mathrm{O}(n)}$ ( альное пространство Березина), состоящее из $\mathrm{O}(n)$-инвариантных функций.

Понятно, что элементы пространства $V_{\theta}^{\mathrm{O}(n)}$ имеют вид

$$
g\left(z_{1}^{2}+\cdots+z_{n}^{2}\right)=\sum c_{p}\left(z_{1}^{2}+\cdots+z_{n}^{2}\right)^{p}
$$

Поэтому $V_{\theta}^{\mathrm{O}(n)}$ можно рассматривать как пространство голоморфных функций от переменной

$$
u=z_{1}^{2}+\cdots+z_{n}^{2}
$$

лежащей в круге $|u|<1$.

ЛЕМма 3.1. Векторы $u^{p}$ образуют ортогональный базис в пространстве $V_{\theta}^{\mathrm{O}(n)}$, причем

$$
\left\|u^{p}\right\|^{2}=\frac{p !(n / 2)_{p}}{(\theta / 2)_{p}(\theta / 2+1 / 2)_{p}} .
$$

ДокАЗАТЕЛЬСтво. Ясно, что векторы $u^{p}$ попарно ортогональны, поэтому нам достаточно вычислить $\left\|u^{p}\right\|^{2}$. Используя соотношения ортогональности (3.4), получаем

$$
\begin{aligned}
\left\|u^{p}\right\|^{2}=\left\|\left(z_{1}^{2}+\cdots+z_{n}^{2}\right)^{p}\right\|^{2} & =\sum_{k_{1} \geqslant 0, \ldots, k_{n} \geqslant 0, \sum k_{j}=p}\left(\frac{p !}{k_{1} ! \cdots k_{n} !}\right)^{2}\left\|z_{1}^{2 k_{1}} \cdots z_{n}^{2 k_{n}}\right\|^{2} \\
& =\sum_{k_{1} \geqslant 0, \ldots, k_{n} \geqslant 0, \sum k_{j}=p}\left(\frac{p !}{k_{1} ! \cdots k_{n} !}\right)^{2} \frac{2 k_{1} ! \cdots 2 k_{n} !}{(\theta)_{2 p}}
\end{aligned}
$$

Нам достаточно вычислить

$$
\sum_{k_{1} \geqslant 0, \ldots, k_{n} \geqslant 0, \sum k_{j}=p} \frac{2 k_{1} ! \cdots 2 k_{n} !}{\left(k_{1} ! \cdots k_{n} !\right)^{2}} .
$$

Обозначим слагаемое этой суммы через $A_{k_{1}, \ldots, k_{n}}$. Рассмотрим производящую функцию

$$
\begin{aligned}
h\left(y_{1}, \ldots, y_{n}\right): & =\sum_{k_{1} \geqslant 0, \ldots, k_{n} \geqslant 0} A_{k_{1}, \ldots, k_{n}} y_{1}^{k_{1}} \cdots y_{n}^{k_{n}} \\
& =\prod_{m=1}^{n} \sum_{k=0}^{\infty} \frac{2 k !}{k ! k !} y_{m}^{k}=\prod_{m=1}^{n}\left(1-4 y_{m}\right)^{-1 / 2}
\end{aligned}
$$

Выражение (3.9) есть коэффициент при $y^{p}$ в равенстве

$$
h(y, \ldots, y)=(1-4 y)^{-n / 2},
$$

и это дает искомьй результат.

Пространства, имитируюшие $V_{\theta}^{\mathrm{O}(n)}$, введены ниже в п. 5.1. 
3.7. Задача Эрстеда. Пусть $b, c$ по-прежнему задаются формулами (1.12). При $\theta>b$ представление $\mathrm{U}(1, n ; \mathbb{K})$ в $H^{\circ}\left[L_{\theta}\right]$ эквивалентно представлению в $L^{2}\left(\mathrm{~B}_{n}(\mathbb{K})\right)$. Естественно возникает следующий вопрос:

можно ли написать явно унитарный сплетающий оператор

$$
S: L^{2}\left(\mathrm{~B}_{n}(\mathbb{K})\right) \rightarrow H^{\circ}\left[L_{\theta}\right] ?
$$

Легко написать общий вид такого оператора. А именно $S$ есть произведение трех операторов

$$
S=\text { const } \cdot R_{\theta}^{-1} M A
$$

где $A$ задан формулой (1.9), $R_{\theta}$ задан формулой (3.6), а оператор

$$
M: L^{2}\left(\mathbb{R}_{+},\left|\frac{\Gamma(b+i s) \Gamma(c+i s)}{\Gamma(2 i s)}\right|^{2}\right) \rightarrow L^{2}\left(\mathbb{R}_{+},\left|\frac{\Gamma(\theta-b+i s) \Gamma(b+i s) \Gamma(c+i s)}{\Gamma(2 i s)}\right|^{2}\right)
$$

есть оператор деления на функцию $\psi(s)$, удовлетворяющую условию

$$
|\psi(s)|=|\Gamma(\theta-b+i s)|
$$

Лемма 3.2. Oператор $S$ задается формулой

$$
S f(z)=\int_{\mathrm{B}_{n}(\mathbb{K})} \Lambda\left(\frac{|z-u|^{2}}{\left(1-|z|^{2}\right)\left(1-|u|^{2}\right)}\right) f(u) d m(u)
$$

где $d$ - инвариантная мера на шаре, а

$$
\Lambda(x)=\mathrm{const} \cdot \int_{0}^{\infty} \overline{\psi(s)}\left|\frac{\Gamma(b+i s) \Gamma(c+i s)}{\Gamma(2 i s)}\right|_{2}^{2} F_{1}(b+i s, b-i s ; b+c ;-x) d s .
$$

ДокАЗАтЕльство. Рассмотрим функцию, равную единице, из пространства $V_{\theta}$. Ее прообраз относительно оператора $S$ легко вычисляется и задается формулой (3.10), где $x=|z|^{2} /\left(1-|z|^{2}\right)$. В силу воспроизводящего свойства для любого элемента $g \in V_{\theta}$ выполнено $\langle g, 1\rangle=g(0)$. В силу унитарности оператора $S$ для любой функции $f \in L^{2}$ выполнено

$$
S f(0)=\int_{\mathrm{B}_{n}(\mathbb{K})} \Lambda\left(\frac{|z|^{2}}{\left.1-|z|^{2}\right)}\right) d m(z)
$$

Теперь ядро оператора $S$ восстанавливается из соображений инвариантности.

Встает вопрос о том, можно ли подобрать $\psi(s)$ так, чтобы интеграл (3.10) можно было явно вычислить. Многолетние попытки автора сделать это были безуспешны, мы склонны думать, что это невозможно. По-видимому, из всех возможных функций $\psi$ простейший вариант

$$
\psi(s)=\Gamma(\theta-b-i s)
$$


является наилучшим.

В $§ 6$ мы пытаемся понять, следует ли полученный интеграл вида (3.10) считать новой спецфункцией.

Есть "метафизический” довод, заставляющий автора склоняться к положительному ответу на этот вопрос. Пусть, например, $\mathbb{K}=\mathbb{R}$. Тогда в пространстве $V_{\theta}$ действует группа $\mathrm{U}(1, n)$, бо́льшая, чем группа $\mathrm{O}(1, n)$, действуюшая в $L^{2}\left(\mathrm{~B}_{n}(\mathbb{R})\right)$. Отождествляя $L^{2}$ с $V_{\theta}$, мы “заставляем" действовать группу $\mathrm{U}(1, n)$ и в $L^{2}\left(\mathrm{~B}_{n}(\mathbb{R})\right)$. Естественно думать, что увеличение групшы симметрий должно повлечь нетривиальный анализ на уровне спецфункций.

Другое обстоятельство, кажущееся автору приятным, - то, что для трех серий полупростых групп $\mathrm{O}(n, \mathbb{C}), \mathrm{Sp}(n, \mathbb{C}), \mathrm{U}(p, q)$ соответствуюшая $\Lambda$-функция выражается как определитель, составленный из $\Lambda$-функций, связанных с пространствами ранга 1, см. $\S 8$.

\section{§4. Многочлены Хана: предварительные сведения}

4.1. Определение. Пусть $a, b, c>0$. Непрерывные двойственные многочлень Хана (см. [30], [31]) определяются формулой

$$
S_{n}\left(s^{2} ; a, b, c\right)=(a+b)_{n}(a+c)_{n 3} F_{2}\left[\begin{array}{c}
-n, a+i s, a-i s \\
a+b, a+c
\end{array} ; 1\right] .
$$

Очевидно, выражение $S_{n}\left(s^{2} ; a, b, c\right)$ не меняется при перестановке параметров $b, c$. Из формулы Куммера (см. [30; следствие 3.3.5])

$$
{ }_{3} F_{2}\left[\begin{array}{c}
\alpha, \beta, \gamma \\
\delta, \varepsilon
\end{array} ; 1\right]=\frac{\Gamma(\varepsilon) \Gamma(\delta+\varepsilon-\alpha-\beta-\gamma)}{\Gamma(\varepsilon-\alpha) \Gamma(\delta+\varepsilon-\beta-\gamma)}{ }_{3} F_{2}\left[\begin{array}{c}
\alpha, \delta-\beta, \delta-\gamma \\
\delta, \delta+\varepsilon-\beta-\gamma)
\end{array} ; 1\right]
$$

вытекает, что $S_{n}\left(s^{2} ; a, b, c\right)$ не меняется при произвольных перестановках $a, b, c$.

4.2. Соотношения ортогональности. Многочлены Хана являются ортогональным базисом в $L^{2}$ на полупрямой с весом

при этом

$$
\left|\frac{\Gamma(a+i s) \Gamma(b+i s) \Gamma(c+i s)}{\Gamma(2 i s)}\right|^{2}
$$

$$
\begin{gathered}
\frac{1}{\pi} \int_{0}^{\infty}\left|\frac{\Gamma(a+i s) \Gamma(b+i s) \Gamma(c+i s)}{\Gamma(2 i s)}\right|^{2} S_{n}\left(s^{2} ; a, b, c\right) S_{m}\left(s^{2} ; a, b, c\right) d x \\
=\Gamma(a+b+n) \Gamma(a+c+n) \Gamma(b+c+n) n ! \delta_{m, n} .
\end{gathered}
$$

4.3. Разностные уравнения. Определим разностный оператор

$$
\mathscr{L} y(s)=B(s) y(s+i)-(B(s)+D(s)) y(s)+D(s) y(s-i),
$$

где

$$
\begin{aligned}
& B(s)=\frac{(a-i s)(b-i s)(c-i s)}{(-2 i s)(1-2 i s)}, \\
& D(s)=\frac{(a+i s)(b+i s)(c+i s)}{(+2 i s)(1+2 i s)} .
\end{aligned}
$$

Многочлены Хана суть собственные функции этого оператора, а именно

$$
\mathscr{L} S_{n}\left(s^{2} ; a, b, c\right)=n \cdot S_{n}\left(s^{2} ; a, b, c\right) .
$$




\section{4. Индексное преобразование и многочлены Хана.}

ЛЕмма 4.1. Образ функции $(1+x)^{-a-b}$ при преобразовании $J_{b, c}$ равен

$$
\frac{\Gamma(a-i s) \Gamma(a+i s)}{\Gamma(a+b) \Gamma(a+c)} .
$$

ДокАЗАТЕЛьство. Лемма сводится к табличному интегралу [32; 7.51.10].

ЛЕмма 4.2. Образ функиии

$$
\left(\frac{x}{x+1}\right)^{n}(1+x)^{-a-b}
$$

при преобразовании $J_{b, c}$ равен

$$
\begin{aligned}
& \frac{\Gamma(a-i s) \Gamma(a+i s)}{\Gamma(a+b) \Gamma(a+c)}{ }_{3} F_{2}\left[\begin{array}{c}
-n, a+i s, a-i s \\
a+b, a+c
\end{array} ; 1\right] \\
& =\frac{|\Gamma(a+i s)|^{2}}{\Gamma(a+b+n) \Gamma(a+c+n)} S_{n}\left(s^{2} ; a, b, c\right) .
\end{aligned}
$$

ДокАЗАТЕЛЬСтво. Нам нужно вычислить образ функции

$$
\begin{aligned}
\left(\frac{x}{x+1}\right)^{n}(1+x)^{-a-b} & =\left(\frac{1+x-1}{x+1}\right)^{n}(1+x)^{-a-b} \\
& =\sum_{k=0}^{n} C_{n}^{k}(-1)^{n-k}(1+x)^{-a-c-n+k}
\end{aligned}
$$

Но образы функций $(1+x)^{-a-c-n+k}$ нами уже вычислены.

\section{§5. Нестандартные формулы Планшереля}

5.1. Пространства $H_{b, c}^{a}$. Пусть положительные числа $a, b, c$ удовлетворяют условиям

$$
a>b, \quad a>c, \quad 2 a>1 .
$$

Рассмотрим пространство $W^{a}$ голоморфных функций в круге $|z|<1$, удовлетворяющих условию

$$
\iint_{|z|<1}|f(z)|^{2}\left(1-|z|^{2}\right)^{2 a-2} d z<\infty
$$

где через $d z$ обозначена мера Лебега в круге.

Введем в пространстве $W^{a}$ скалярное произведение по формуле

$$
\langle f, g\rangle=\frac{1}{\pi \Gamma(2 a-1)} \iint_{|z|<1} f(z) \overline{g(z)}\left(1-|z|^{2}\right)^{2 a-2}{ }_{2} F_{1}\left(a-b, a-c ; 2 a-1 ; 1-|z|^{2}\right) d z .
$$

Полученное гильбертово пространство мы обозначим через $W_{b, c}^{a}$. 
ЗАМЕчАнИЕ. По запасу функций пространство $W_{b, c}^{a}$ не зависит от $b, c$ и совпадает с $W^{a}$.

ЛЕМма 5.1. Функиии $z^{k}$ образуют в $W_{b, c}^{a}$ ортогональный базис, причем

$$
\left\langle z^{k}, z^{k}\right\rangle_{W_{b, c}^{a}}=\frac{k ! \Gamma(b+c+k)}{\Gamma(a+b+k) \Gamma(a+c+k)} .
$$

ДокАЗАТЕльство. Ортогональность функций $z^{k}$ очевидна, вычислим

$$
\begin{aligned}
\left\langle z^{k}, z^{k}\right\rangle & =\frac{1}{\pi \Gamma(2 a-1)} \iint_{|z|<1}|z|^{2 k}\left(1-|z|^{2}\right)^{2 a-2}{ }_{2} F_{1}\left(a-b, a-c ; 2 a-1 ; 1-|z|^{2}\right) d z \\
& =\frac{2}{\Gamma(2 a-1)} \int_{0}^{1} r^{2 k+1}\left(1-r^{2}\right)^{2 a-2}{ }_{2} F_{1}\left(a-b, a-c ; 2 a-1 ; 1-r^{2}\right) d r \\
& =\frac{1}{\Gamma(2 a-1)} \int_{0}^{1} y^{k}(1-y)^{2 a-2}{ }_{2} F_{1}(a-b, a-c ; 2 a-1 ; 1-y) d y \\
& =\frac{1}{\Gamma(2 a-1)} \int_{0}^{1}(1-v)^{k} v^{2 a-2}{ }_{2} F_{1}(a-b, a-c ; 2 a-1 ; v) d v .
\end{aligned}
$$

Далее все сводится к табличному интегралу [32; 7.512.4].

ЗАмЕчАнИЕ. Мы видим, что радиальные пространства Березина $V_{\theta}^{\mathrm{O}(n)}$, введенные выше в п. 3.6, являются частным случаем пространств $W_{b, c}^{a}$ при

$$
b=\frac{n}{4}-\frac{1}{4}, \quad c=\frac{n}{4}+\frac{1}{4}, \quad a=\theta-\frac{n}{4}+\frac{1}{4} .
$$

\section{2. Воспроизводящее ядро пространства $W_{b, c}^{a}$.}

ЛЕмма 5.2. Воспроизводящее ядро пространства $W_{b, c}^{a}$ равно

$$
K_{b, c}^{a}(z, u)=\frac{\Gamma(a+b) \Gamma(a+c)}{\Gamma(b+c)}{ }_{2} F_{1}\left[\begin{array}{c}
a+b, a+c \\
b+c
\end{array} ; z \bar{u}\right]
$$

ДокаЗАТЕЛьство очевидно. Действительно, положим $\varphi_{w}(z)=K_{b, c}^{a}(z, w)$. Тогда для любой голоморфной функции $f(z)=\sum_{k=0}^{\infty} c_{k} z^{k}$ выполнено

$$
\begin{aligned}
\left\langle f, \varphi_{w}\right\rangle_{W_{b, c}^{a}} & =\sum_{k=0}^{\infty} c_{k} w^{k} \frac{\Gamma(a+b+k) \Gamma(a+c+k)}{k ! \Gamma(b+c+k)}\left\langle z^{k}, z^{k}\right\rangle \\
& =\sum_{k=0}^{\infty} c_{k} w^{k}=f(w) .
\end{aligned}
$$

ЗАмЕчАнИЕ. Ядра $K_{b, c}^{a}(z, u)$ являются частными случаями ядер, рассматривавшихся Гроссом и Ричардсом [33], а также частными случаями ядер, изучавшихся Оджиевичем [34]. 
5.3. Формула Планшереля. Пусть $g \in W_{b, c}^{a}$. Рассмотрим преобразование

$$
\begin{aligned}
J_{b, c}^{a} g(s)= & \frac{1}{|\Gamma(a+i s)|^{2} \Gamma(b+c)} \int_{0}^{\infty}(1+x)^{-a-b} g\left(\frac{x}{x+1}\right) \\
& \times{ }_{2} F_{1}(b+i s, b-i s ; b+c ;-x) x^{b+c-1}(1+x)^{b-c} d x .
\end{aligned}
$$

ТЕОРема 5.3. Oператор $J_{b, c}^{a}$ является унитарным оператором

$$
W_{b, c}^{a} \rightarrow L^{2}\left(\mathbb{R}_{+},\left|\frac{\Gamma(a+i s) \Gamma(b+i s) \Gamma(c+i s)}{\Gamma(2 i s)}\right|^{2}\right)
$$

ДокАЗАТЕЛьСтво. Образ функции $f(z)=z^{k}$ при нашем преобразовании есть

$$
\frac{S_{n}\left(s^{2} ; a, b, c\right)}{\Gamma(a+b+k) \Gamma(a+c+k)},
$$

и теперь унитарность оператора следует из формулы (5.2) и соотношений ортогональности для многочленов Хана.

5.4. Оператор $d / d z$. Обозначим через $W^{\infty}$ пространство функций, голоморфных в круге $|z|<1$ и гладких вплоть до границы. Пусть $a>1$. Тогда согласно лемме 1.1 для $f \in W^{\infty}$ функция $J_{b, c}^{a} f$ голоморфна в полосе $|\operatorname{Im} s|<1+\varepsilon$.

ПРЕДЛОЖЕНИЕ 5.4. Для любой $f \in W^{\infty}$ и любого $а>1$ выполнено

$$
J_{b, c}^{a} \frac{d}{d z} f=\mathscr{L} J_{b, c}^{a} f
$$

где $\mathscr{L}$ - разностный оператор, заданньй формулой (4.1).

ДокАЗАТЕЛЬство. Образ функций $z^{k}$ задается формулой (5.3), а многочлены Хана удовлетворяют разностным уравнениям (4.2).

\section{§ 6. $\Lambda$-функция и ее свойства}

Мотивировка для введения функции, рассматриваемой в этом параграфе, содержится выше в п. 3.7.

6.1. Определение. Пусть $a, b, c \in \mathbb{R}, x \in \mathbb{R}_{+}$. Мы определяем $\Lambda$-функцию по формуле

$$
\begin{aligned}
\Lambda_{b, c}^{a}(x)= & \frac{1}{\pi \Gamma(b+c)} \int_{0}^{\infty} \Gamma(a+i s) \frac{\Gamma(b+i s) \Gamma(b-i s) \Gamma(c+i s) \Gamma(c-i s)}{\Gamma(2 i s) \Gamma(-2 i s)} \\
& \times{ }_{2} F_{1}(b+i s, b-i s ; b+c ;-x) d s .
\end{aligned}
$$


ЗАмЕчАнИЕ. Функция $\Lambda_{b, c}^{a}(x)$ продолжается по голоморфности в область $\operatorname{Re} a$, $\operatorname{Re} b, \operatorname{Re} c>0$. Нам будет приятнее формулировать ее свойства для вещественных $a, b, c$.

Нам не удалось выразить интеграл (6.1) через стандартные специальные функции (см. список в [11]) с помощю конечного числа алгебраических операций, за исключением некоторых случаев с выделенными значениями $b, c$ (см. ниже п. 6.6). Мы также склонны думать, что это невозможно. Нетривиальность интеграла (6.1) состоит не столько в том, что подыптегральное выражение несколько замысловато, сколько в необычных пределах интегрирования. Действительно, если при вещественных $a, b, c$ заменить пределы интегрирования на

$$
\int_{-\infty}^{+\infty}
$$

что равносильно взятию

$$
\operatorname{Re} \Lambda_{b, c}^{a}(x)
$$

то наш интеграл преврашается в интеграл типа Слейтер (см. [35], [36]), и стандартная техника позволяет выписывать для $\operatorname{Re} \Lambda_{b, c}^{a}(x)$ различные представления в виде рядов. Автору, однако, не удалось на этом пути получить радующего глаз окончательного выражения для $\operatorname{Re} \Lambda_{b, c}^{a}(x)$.

При некоторых значениях $b, c$ выражение (6.3) может быть вычислено явно (см. п. 6.7).

Если мы подставим $x=0$ в (6.3), то получим барнсовский интеграл

$$
\operatorname{Re} \Lambda_{b, c}^{a}(0)=\frac{1}{2 \pi \Gamma(b+c)} \int_{-\infty}^{+\infty} \frac{\Gamma(a+i s) \Gamma(b+i s) \Gamma(b-i s) \Gamma(c+i s) \Gamma(c-i s)}{\Gamma(2 i s) \Gamma(-2 i s)} d s
$$

которьй с помощью обычного метода Барнса (см. [35], [36], [30]) представляется виде суммы двух гипергеометрических рядов типа ${ }_{4} F_{4}(1)$ :

$$
\begin{aligned}
\operatorname{Re} \Lambda_{b, c}^{a}(0)= & \sum_{0}^{\infty} \frac{(-1)^{n} \Gamma(a-b-n) \Gamma(2 b+n) \Gamma(c-b-n) \Gamma(c+b+n)}{n ! \Gamma(2 b+2 n) \Gamma(-2 b-2 n)} \\
& +\sum_{0}^{\infty} \frac{(-1)^{n} \Gamma(a-c-n) \Gamma(2 c+n) \Gamma(b-c-n) \Gamma(b+c+n)}{n ! \Gamma(2 c+2 n) \Gamma(-2 c-2 n)} .
\end{aligned}
$$

Мы видим, что $\Lambda_{b, c}^{a}(0)$ является чем-то вроде барнсовского интеграла по незамкнутому контуру. Тем не менее, выражение $\Lambda_{b, c}^{a}(0)$ все же “лучше" неопределенного барнсовского интеграла

$$
\frac{1}{2 \pi \Gamma(b+c)} \int_{u}^{+\infty} \Gamma(a+i s) \frac{\Gamma(b+i s) \Gamma(b-i s) \Gamma(c+i s) \Gamma(c-i s)}{\Gamma(2 i s) \Gamma(-2 i s)} d s
$$

так как точка $s=0$ является выделенной точкой для второго сомножителя подынтегрального выражения. 
6.2. Имитация задачи Эрстеда. Мы рассматриваем пространство $W_{b, c}^{a}$. Оператор $J_{b, c}^{a}$ переводит его унитарно в

$$
L^{2}\left(\mathbb{R}_{+},\left|\frac{\Gamma(a+i s) \Gamma(b+i s) \Gamma(c+i s)}{\Gamma(2 i s)}\right|^{2} d s\right) .
$$

Далее оператор умножения

$$
M g(s)=\Gamma(a+i s) g(s)
$$

переводит унитарно пространство (6.5) в пространство

$$
L^{2}\left(\mathbb{R}_{+},\left|\frac{\Gamma(b+i s) \Gamma(c+i s)}{\Gamma(2 i s)}\right|^{2} d s\right)
$$

а обратное индексное преобразование $\left(J_{b, c}\right)^{-1}$ переводит унитарно $(6.6)$ в

$$
L^{2}\left(\mathbb{R}_{+}, x^{b+c-1}(1+x)^{b-c} d x\right)
$$

Таким образом, мы получаем унитарный оператор

$$
\left(J_{b, c}\right)^{-1} \circ M \circ J_{b, c}^{a}: W_{b, c}^{a} \rightarrow L^{2}\left(\mathbb{R}_{+}, x^{b+c-1}(1+x)^{b-c} d x\right)
$$

Этот оператор и есть имитация оператора

$$
V_{\theta} \rightarrow L^{2}\left(\mathrm{~B}_{m}(\mathbb{K})\right)
$$

обсуждавшегося в п. 3.7. Функция $\Lambda_{b, c}^{a}$ есть образ функции $f(z)=1$ при этом отображении.

6.3. Прямые следствия определения $\Lambda$-функции. Формула обращения для индексного преобразования влечет

$\frac{1}{\Gamma(b+c)} \int_{0}^{\infty} \Lambda_{b, c}^{a}(x){ }_{2} F_{1}(b+i s, b-i s ; b+c ;-x) x^{b+c-1}(1+x)^{b-c} d x=\Gamma(a+i s)$

Из формулы Планшереля для индексного преобразования следует

$$
\begin{aligned}
\int_{0}^{\infty} \Lambda_{b, c}^{a}(x) \overline{\Lambda_{b, c}^{a}(x)} x^{b+c-1}(1+x)^{b-c} d x & =\frac{1}{\pi} \int_{0}^{\infty}\left|\frac{\Gamma(a+i s) \Gamma(b+i s) \Gamma(c+i s)}{\Gamma(2 i s)}\right|^{2} d s \\
& =\Gamma(a+b) \Gamma(a+c) \Gamma(b+c)
\end{aligned}
$$

(по поводу последней строчки см. [30; 3.6]). 
6.4. Дифференциально-разностные уравнения. Тождество

$$
{ }_{2} F_{1}(\alpha, \beta ; \gamma ;-x)=(1+x)^{\gamma-\alpha-\beta}{ }_{2} F_{1}(\gamma-\alpha, \gamma-\beta ; \gamma ;-x)
$$

(см. [29; том 1, 2.1(23)]) влечет

$$
\Lambda_{b, c}^{a}(x)=(1+x)^{c-b} \Lambda_{c, b}^{a}(x) .
$$

Дифференцируя интеграл (6.1) по параметру $x$ с помошью формулы

$$
\frac{d}{d x}{ }_{2} F_{1}(\alpha, \beta ; \gamma ; x)=\frac{\alpha \beta}{\gamma}{ }_{2} F_{1}(\alpha+1, \beta+1 ; \gamma+1 ; x),
$$

получаем

$$
\begin{aligned}
\frac{d}{d x} \Lambda_{b, c}^{a}(x)= & -\frac{1}{\Gamma(b+c+1)} \int_{0}^{\infty} \Gamma(a+i s)\left|\frac{\Gamma(b+1+i s) \Gamma(c+i s)}{\Gamma(2 i s)}\right|^{2} \\
& \times{ }_{2} F_{1}(b+1+i s, b+1-i s ; b+c+1 ;-x) d s=-\Lambda_{b+1, c}^{a}(x)
\end{aligned}
$$

и окончательно

$$
\frac{d}{d x} \Lambda_{b, c}^{a}(x)=-\Lambda_{b+1, c}^{a}(x) .
$$

Отметим следствие из формул (6.7), (6.8):

$$
\Lambda_{b+k, c+l}^{a}(x)=(-1)^{k+l}(1+x)^{c+l-b-k} \frac{d^{l}}{d x^{l}}(1+x)^{b+k-c} \frac{d^{k}}{d x^{k}} \Lambda_{b, c}^{a}(x) .
$$

Используя формулу (см. [30; 2.5.7])

$$
\left(x \frac{d}{d x}+\gamma-1\right){ }_{2} F_{1}(\alpha, \beta ; \gamma ; x)=(\gamma-1){ }_{2} F_{1}(\alpha, \beta ; \gamma-1 ; x),
$$

мы получаем

$$
\begin{aligned}
&\left(x \frac{d}{d x}+b+c-1\right) \Lambda_{b, c}^{a}(x) \\
&=- \frac{1}{\Gamma(b+c-1)} \int_{0}^{\infty} \Gamma(a+i s)\left|\frac{\Gamma(b+i s) \Gamma(c-1+i s)}{\Gamma(2 i s)}\right|^{2}(c+i s)(c-i s) \\
& \quad \times{ }_{2} F_{1}(b+i s, b-i s ; b+(c-1) ;-x) d s .
\end{aligned}
$$

Далее, представляем $(c+i s)(c-i s)$ в виде

$$
(c+i s)(c-i s)=\left(c^{2}-a^{2}\right)+(2 a+1)(a+i s)-(a+i s)(a+1+i s)
$$

и получаем

$$
\left(x \frac{d}{d x}+b+c-1\right) \Lambda_{b, c}^{a}(x)=\left(c^{2}-a^{2}\right) \Lambda_{b, c}^{a}(x)+(2 a+1) \Lambda_{b, c}^{a+1}(x)-\Lambda_{b, c}^{a+2}(x)
$$

или

$$
-x \Lambda_{b+1, c}^{a}(x)=\left(c^{2}-a^{2}-b-c+1\right) \Lambda_{b, c}^{a}(x)+(2 a+1) \Lambda_{b, c}^{a+1}(x)-\Lambda_{b, c}^{a+2}(x) .
$$

Приведенный нами список разностных уравнений заведомо неполон. 


\section{5. Один интеграл.}

ПРЕДЛОЖЕНИЕ 6.1. Для $n=0,1,2, \ldots$

$$
\begin{aligned}
& 2 \operatorname{Re} \int_{0}^{\infty} \Lambda_{b, c}^{a}(x) \overline{\Lambda_{b, c}^{a+n}(x)} x^{b+c-1}(1+x)^{b-c} d x \\
& =(2 a)_{2 n} \Gamma(a+c) \Gamma(b+c) \Gamma(a+b)_{4} F_{3}\left[\begin{array}{c}
-n / 2,-(n+1) / 2, a+b, a+c \\
-n+1,2 a, 2 a+1
\end{array}\right] .
\end{aligned}
$$

Отметим, что выражение ${ }_{4} F_{3}$ в нашем случае является не рядом, а конечной суммой. Правую часть можно также переписать в форме

$$
\begin{aligned}
& \Gamma(a+b) \Gamma(a+c) \Gamma(b+c)(2 a)_{2 n} \\
& -n \Gamma(a+b+1) \Gamma(a+c+1) \Gamma(b+c)(2 a+2)_{2 n-2}+n \Gamma(b+c) \\
& \quad \times \sum_{k=2}^{[(n+1) / 2]} \frac{(n-k-1) \cdots(n-2 k+1)}{k !} \Gamma(a+b+k) \Gamma(a+c+k)(2 a+2 k)_{2 n-2 k} .
\end{aligned}
$$

ДокАЗАТЕльство. В силу формулы Планшереля наш интеграл равен

$$
\begin{aligned}
& \frac{2}{\pi} \int_{0}^{\infty} \operatorname{Re}[\Gamma(a+i s) \overline{\Gamma(a+i s+n)}]\left|\frac{\Gamma(b+i s) \Gamma(c+i s)}{\Gamma(2 i s)}\right|^{2} d s \\
& \quad=\frac{1}{\pi} \int_{0}^{\infty}\left[(a+i s)_{n}+(a-i s)_{n}\right]\left|\frac{\Gamma(a+i s) \Gamma(b+i s) \Gamma(c+i s)}{\Gamma(2 i s)}\right|^{2} d s .
\end{aligned}
$$

ЛЕММА 6.2 .

$$
\begin{aligned}
& (a+i s)_{n}+(a-i s)_{n} \\
& =(2 a)_{2 n}-n(2 a+2)_{2 n-2}(a+i s)(a-i s) \\
& \quad+n \sum_{k=2}^{[(n+1) / 2]} \frac{(-1)^{k}(-n+k+1)_{k-1}}{k !}(2 a+2 k)_{2 n-2 k}(a+i s)_{k}(a-i s)_{k} \\
& =(2 a)_{2 n} \sum_{j=0}^{[(n+1) / 2]} \frac{(-n / 2)_{j}(-(n+1) / 2)_{j}(a-i s)_{j}(a+i s)_{j}}{j !(-n+1)_{j}(2 a)_{2 j}}
\end{aligned}
$$

С помощью леммы 6.2 интеграл (6.13) переписьвается в виде

$$
\begin{aligned}
(2 a)_{2 n} & \sum_{k=0}^{[(n+1) / 2]} \frac{(-n / 2)_{j}(-(n+1) / 2)_{j}}{j !(-n+1)_{j}(2 a)_{2 j}} \\
& \times \int_{0}^{\infty}\left|\frac{\Gamma(a+j+i s) \Gamma(b+i s) \Gamma(c+i s)}{\Gamma(2 i s)}\right|^{2} d s \\
= & (2 a)_{2 n} \sum_{k=0}^{[(n+1) / 2]} \frac{(-n / 2)_{j}(-(n+1) / 2)_{j}}{j !(-n+1)_{j}(2 a)_{2 j}} \Gamma(b+c) \Gamma(a+j+b) \Gamma(a+j+c),
\end{aligned}
$$

что и влечет искомый результат. 
6.6. Связь с $\lambda$-функцией. Функция $\lambda(z, a)$ задается формулой

$$
\lambda(z, a):=\int_{0}^{a} z^{-t} \Gamma(t+1) d t .
$$

Нам будет удобней переобозначить ее как

$$
\lambda^{*}(z, b)=\int_{b}^{i \infty} z^{-t} \Gamma(t+1) d t
$$

(это тот же неопределенный интеграл с другой начальной точкой). Функция $\lambda$ входит в довольно экзотическое семейство спецфункций (иногда они называются функииями типа Вольтерра), включающее также

$$
\begin{aligned}
\mu(z, p) & =\int_{0}^{\infty} \frac{t^{p} z^{t} d t}{\Gamma(t+1)} ; \mu(z, p, \rho)=\int_{0}^{\infty} \frac{t^{p} z^{t+\rho} d t}{\Gamma(\rho+t+1)} \\
\nu(z) & =\int_{0}^{\infty} \frac{z^{t} d t}{\Gamma(t+1)} ; \quad \nu(z, \rho)=\int_{0}^{\infty} \frac{z^{t+\rho} d t}{\Gamma(\rho+t+1)}
\end{aligned}
$$

Отметим некоторое внешнее сходство этих функций с (6.1) - это интегралы, напоминающие барнсовские, но имеющие "неправильные" пределы интегрирования. Функции $\mu(z, p), \mu(z, p, \rho)$ появляются у Вольтерра [37] в связи с дробными дифференцированиями с логарифмическими добавками (см. также [10]). Далее функции $\mu, \nu$ оживленно обсуждались во французских и бельгийских математических журналах первой половины 40-х годов XX века (Humbert, Poli, Colombo, Parodi и др., см., например, [38], [39]). Первое упоминание о функции $\lambda$, которое мне удалось найти, содержится в таблицах Мак-Лахлана, Гумберта, Поли [40].

Теория функций $\nu, \mu$ излагается в [29; 18.3] (там же есть большая библиография), интегралы с функциями $\nu, \mu, \lambda$ можно найти в таблицах Прудникова, Брычкова, Маричева, см. [11; соответствующие разделы в томах 3-5].

Сейчас мы покажем, что функции

$$
\Lambda_{1 / 2+k, l}^{a}(x), \quad \Lambda_{k, 1 / 2+l}^{a}(x)
$$

выражаются через функцию $\lambda^{*}(z, b)$ (в частности это так для $\Lambda$-функций, связанных с группами подсерии $\mathrm{O}(1,2 n+1))$. Рассмотрим, например, функцию

$$
\begin{aligned}
\Lambda_{1 / 2,1}^{a}(x):= & \frac{1}{\pi \Gamma(3 / 2)} \int_{0}^{\infty} \Gamma(a+i s)\left|\frac{\Gamma(1 / 2+i s) \Gamma(1+i s)}{\Gamma(2 i s)}\right|^{2} \frac{1}{2 s \sqrt{x}} \sin (2 s \operatorname{arcsh} \sqrt{x}) d s \\
= & -\frac{4}{\sqrt{\pi x}} \int_{0}^{\infty} \Gamma(a+i s) i s\left[e^{2 i s \operatorname{arcsh} \sqrt{x}}-e^{-2 i s \operatorname{arcsh} \sqrt{x}}\right] d s \\
= & -\frac{4}{\sqrt{\pi x}} \int_{0}^{\infty}(\Gamma(a+1+i s)-a \Gamma(a+i s)) \\
& \times\left[\left(\sqrt{x^{2}+1}+x\right)^{2 i s}-\left(\sqrt{x^{2}+1}-x\right)^{2 i s}\right] d s,
\end{aligned}
$$

и мы получаем сумму четырех интегралов, выражаемых через функцию $\lambda^{*}$. 
Далее, применяя формулу (6.9), мы получаем выражение для $\Lambda_{1 / 2+k, 1+l}^{a}(x)$ через производные от функции $\lambda^{*}$. Случай функций $\Lambda_{k, 1 / 2+l}^{a}$ аналогичен.

Отметим, что производные от функции $\lambda^{*}$ выражаются через саму функцию $\lambda^{*}$ :

$$
\begin{aligned}
\frac{d}{d z} \lambda^{*}(z, b) & =-\int_{b}^{+i \infty} \frac{t}{z} z^{-t} \Gamma(t+1) d t \\
& =-\frac{1}{z} \int_{b}^{+i \infty}(\Gamma(t+2)-\Gamma(t+1)) z^{-t} d t=-\lambda^{*}(z, b+1)+\frac{1}{z} \lambda^{*}(z, b) .
\end{aligned}
$$

6.7. Явно вычислимые случаи для $\operatorname{Re} \Lambda_{b, c}^{a}$. Пусть $k, l=0,1,2, \ldots$ Покажем, что следуюшие функции

$$
\operatorname{Re} \Lambda_{1 / 2+k, l}^{a}(x), \quad \operatorname{Re} \Lambda_{k, 1 / 2+l}^{a}(x)
$$

могут быть явно вычислены. Вычислим, например,

$$
\begin{aligned}
\operatorname{Re} \Lambda_{1 / 2,1}^{a}:= & \text { const } \int_{-\infty}^{+\infty} \Gamma(a+i s) s^{2} \frac{1}{2 s \sqrt{x}} \sin (2 s \operatorname{arcsh} \sqrt{x}) d s \\
= & \text { const } \int_{-\infty}^{+\infty}(\Gamma(a+1+i s)-a \Gamma(a+i s)) \\
& \times\left[\left(\sqrt{x^{2}+1}+x\right)^{2 i s}-\left(\sqrt{x^{2}+1}-x\right)^{2 i s}\right] d s .
\end{aligned}
$$

Применяя формулу (см., например [30; (2.4.1)])

$$
\frac{1}{2 \pi i} \int_{-i \infty}^{+i \infty} \Gamma(u+t) z^{t} d t=z^{u} e^{-z}
$$

получаем

$$
\begin{aligned}
\text { const }[ & \left(x+\sqrt{x^{2}+1}\right)^{2 a}\left(\left(x+\sqrt{x^{2}+1}\right)^{2}-a\right) \exp \left\{-\left(x+\sqrt{x^{2}+1}\right)^{2}\right\} \\
& \left.-\left(\sqrt{x^{2}+1}-x\right)^{2 a}\left(\left(\sqrt{x^{2}+1}-x\right)^{2}-a\right) \exp \left\{-\left(\sqrt{x^{2}+1}-x\right)^{2}\right\}\right] .
\end{aligned}
$$

Чтобы получить явное выражение для $\operatorname{Re} \Lambda_{1 / 2+k, l}^{a}(x)$, достаточно применить формулу (6.9).

ЗАмЕчАниЕ. Некоторая громоздкость формул этого и предыдушего пункта исчезает, если перейти к новой переменной $t$ по формуле

$$
x=\operatorname{sh}^{2} t .
$$


6.8. Комментарии. Как было объяснено в конце $\S 3$, в выборе формулы для $\Lambda$-функции есть определенный произвол. Некоторые из свойств нашего списка остаются в силе при произвольном выборе функции $\psi$ (см. (3.10)), т.е. для общих интегралов вида

$$
\begin{gathered}
\frac{1}{\pi \Gamma(b+c)} \int_{0}^{\infty} \Gamma(a+i s) e^{i u(s)} \frac{\Gamma(b+i s) \Gamma(b-i s) \Gamma(c+i s) \Gamma(c-i s)}{\Gamma(2 i s) \Gamma(-2 i s)} \\
\times{ }_{2} F_{1}(b+i s, b-i s ; b+c ;-x) d s
\end{gathered}
$$

где $\operatorname{Im} u(s)=0$. Так обстоит дело с интегралами из п. 6.3, дифференциально-разностньми уравнениями (6.7)-(6.9), с теоремой 7.1, предложением 7.2, теоремой 8.1 .

Забавно, однако, что у $\Lambda$-функции есть и ряд свойств, которые не вытекают из мотивировки п. 3.7 и которые верны лишь при нашем выборе функции $\psi$. К этим свойствам относятся разностное уравнение (6.10) и весь материал пп. 6.5-6.7.

Отметим также, что дифференциально-разностные уравнения п. 6.4 и выражения через $\lambda$-функцию остаются в силе для неопределенного слейтеровского интеграла (6.4). Однако никакие другие полученные нами свойства $\Lambda$-функции для неопределенных интегралов не выживают.

\section{§7. Зазеркальные базисы и гипергруппа обобщенного сдвига}

7.1. Зазеркальные базисы. В п. 5.3 нами был определен унитарный оператор

$$
Q=\left(J_{b, c}\right)^{-1} \circ M \circ J_{b, c}^{a}: W_{b, c}^{a} \rightarrow L^{2}\left(\mathbb{R}_{+}, x^{b+c-1}(1+x)^{b-c} d x\right) .
$$

Через

$$
\Xi_{n}(x)=\Xi_{n}(x ; a ; b, c)
$$

мы обозначим образ функции $z^{n}$ при преобразовании $Q$.

ТЕОРема 7.1. а) Система функиий $\Xi_{n}$ образует ортогональный базис в $L^{2}\left(\mathbb{R}_{+}, x^{b+c-1}(1+x)^{b-c} d x\right)$.

b) Функиии $\Xi_{n}$ выражаются через $\Lambda$-функиию по формуле

$$
\begin{aligned}
\Xi_{n}(x ; a, b, c)= & \frac{(b+c)_{n}}{\Gamma(a+c) \Gamma(a+b+n)} \\
& \times \sum_{j=0}^{n} \frac{(-1)^{j}(-n)_{j}}{j !(a+c)_{j}(b+c)_{j}} x^{-b-c+1} \frac{d^{j}}{d x^{j}} x^{b+c+j-1} \Lambda_{b, c+j}^{a} .
\end{aligned}
$$

ДокАЗАтЕльство. В доказательстве нуждается лишь утверждение b). Напомним, что образ функции $z^{n}$ под действием оператора $J_{b, c}^{a}$ является многочленом Хана. Поэтому нам достаточно вычислить интеграл

$$
\begin{aligned}
& \frac{1}{\Gamma(a+b+n) \Gamma(a+c+n) \Gamma(b+c)} \int_{0}^{\infty} \Gamma(a+i s)\left|\frac{\Gamma(b+i s) \Gamma(c+i s)}{\Gamma(2 i s)}\right|^{2} \\
& \quad \times{ }_{2} F_{1}(b+i s, b-i s ; b+c ;-x) S_{n}\left(x^{2} ; a, b, c\right) d s .
\end{aligned}
$$


Воспользовавшись симметрией многочленов Хана по $a, b, c$, мы переписываем этот интеграл в виде

$$
\begin{aligned}
& \frac{1}{\Gamma(a+b+n) \Gamma(a+c+n) \Gamma(b+c)} \int_{0}^{\infty} \Gamma(a+i s)\left|\frac{\Gamma(b+i s) \Gamma(c+i s)}{\Gamma(2 i s)}\right|^{2} \\
& \quad \times{ }_{2} F_{1}(b+i s, b-i s ; b+c ;-x)(a+c)_{n}(b+c)_{n 3} F_{2}\left(\begin{array}{c}
-n, c+i s, c-i s \\
a+c, b+c
\end{array} 1\right) d s .
\end{aligned}
$$

Разлагая ${ }_{3} F_{2}$ в сумму, получаем

$$
\begin{aligned}
& \frac{(b+c)_{n}}{\Gamma(a+b+n) \Gamma(a+c) \Gamma(b+c)} \sum_{j=0}^{n} \frac{(-n)_{j}}{j !(a+c)_{j}(b+c)_{j}} \\
& \quad \times \int_{0}^{\infty} \Gamma(a+i s)\left|\frac{\Gamma(b+i s) \Gamma(c+j+i s)}{\Gamma(2 i s)}\right|_{2}^{2}{ }_{2} F_{1}(b+i s, b-i s ; b+c ;-x) d s .
\end{aligned}
$$

Далее мы применяем формулу [29; том 1, 2.8(22)]

$$
(\mu)_{k} y_{2}^{\mu-1} F_{1}(\alpha, \beta ; \mu ; y)=\frac{d^{k}}{d y^{k}} y^{\mu+k-1}{ }_{2} F_{1}(\alpha, \beta ; \mu+k ; y)
$$

и получаем

$$
\begin{aligned}
& \frac{(b+c)_{n}}{\Gamma(a+b) \Gamma(a+c)} \sum_{j=0}^{n} \frac{(-n)_{j}}{j !(a+c)_{j}(b+c)_{j}} \frac{1}{\Gamma(b+c+j)}(-1)^{j} x^{1-b-c} \frac{d^{j}}{d x^{j}} x^{b+c+j-1} \\
& \quad \times \int_{0}^{\infty} \Gamma(a+i s)\left|\frac{\Gamma(b+i s) \Gamma(c+j+i s)}{\Gamma(2 i s)}\right|_{2}^{2}{ }_{2} F_{1}(b+i s, b-i s ; b+(c+j) ;-x) d s
\end{aligned}
$$

что и дает искомое выражение.

7.2. Операторы обобщенного сдвига. Гипергруппа обобщенного сдвига существенный элемент теории индексного гипергеометрического преобразования (см. [5], [7]). Мы лишь слегка коснемся этого предмета.

Рассмотрим множество $J \subset \mathbb{R}_{+} \times \mathbb{R}_{+} \times \mathbb{R}_{+}$, состоящее из точек $(x, y, z)$ таких, что три числа $\operatorname{arcsh} \sqrt{x}, \operatorname{arcsh} \sqrt{y}, \operatorname{arcsh} \sqrt{z}$ удовлетворяют неравенству треугольника. Рассмотрим функцию $K(x, y, z)$ на $\mathbb{R}_{+} \times \mathbb{R}_{+} \times \mathbb{R}_{+}$, равную нулю тождественно вне множества $J$, а на множестве $J$ равную

$$
\begin{aligned}
K(x, y, z)= & \frac{2^{-4 b} \Gamma(b+c)}{\Gamma(b+c-1 / 2)} \frac{((1+x)(1+y)(1+z))^{c-1}}{(x y z)^{b+c-1}} \\
& \times\left(1-B^{2}\right)^{b+c-1 / 2}{ }_{2} F_{1}\left(2 b-1,2 c-1 ; b+c-\frac{1}{2} ; \frac{1-B}{2}\right),
\end{aligned}
$$

где

$$
B=\frac{x+y+z+2}{2(1+x)(1+y)(1+z)} .
$$

Oператор обобщенного сдвига $T_{z}$ в $L^{2}\left(\mathbb{R}_{+}, x^{b+c-1}(1+x)^{b-c}\right)$ задан формулой

$$
T_{z} f(y)=\int_{0}^{\infty} K(x, y, z) f(x) x^{b+c-1}(1+x)^{b-c} d y .
$$

Известно, что

$$
J_{b, c} T_{z}\left(J_{b, c}\right)^{-1} g(s)={ }_{2} F_{1}(b+i s, b-i s ; b+c ;-z) f(s) .
$$


ПРЕДЛОЖЕНИЕ 7.2 .

$$
\begin{aligned}
\int_{0}^{\infty} & \int_{0}^{\infty} \Lambda_{b, c}^{a}(x) \overline{\Lambda_{b, c}^{a}(x)} K(x, y, z) x^{b+c-1}(1+x)^{b-c} y^{b+c-1}(1+y)^{b-c} d x d y \\
& =\Gamma(a+b) \Gamma(a+c) \Gamma(b+c)(1+z)^{-a-b}
\end{aligned}
$$

ДокАЗАТЕЛЬСтво. Выражение в левой части есть скалярное произведение $\Lambda_{b, c}^{a}$ и $T_{z} \Lambda_{b, c}^{a}$ в $L^{2}\left(\mathbb{R}_{+}, x^{b+c-1}(1+x)^{b-c}\right)$. В силу формулы Планшереля и $(7.1)$ это равно

$$
\int_{0}^{\infty}\left|\frac{\Gamma(b+i s) \Gamma(c+i s)}{\Gamma(2 i s)}\right|^{2} \Gamma(a+i s) \overline{\Gamma(a+i s)}_{2} F_{1}(b+i s, b-i s ; b+c ;-z) d s .
$$

Последнее выражение есть обратное индексное преобразование от $|\Gamma(a+i s)|^{2}$. Но оно нам известно из леммы 4.1 .

7.3. Обобщенный сдвиг и зазеркальные базисы. По-видимому, оператор обобшенного сдвига имеет в зазеркальном базисе простую явно вычислимую матрицу.

Автор умеет вычислять лишь ее первую строку.

ПРЕДЛОЖЕНИЕ 7.3.

$$
T_{z} \Lambda_{b, c}^{a}=T_{z} \Xi_{0}(x ; a, b, c)=(1+z)^{a-b} \sum_{n=0}^{\infty} \frac{1}{n !(b+c)_{n}}\left(\frac{z}{z+1}\right)^{n} \Xi_{n}(x ; a, b, c) .
$$

ДокаЗАТЕЛЬство. Индексное преобразование от $T_{z} \Lambda_{b, c}^{a}$ равно

$$
\begin{aligned}
\Gamma(a+i s) & { }_{2} F_{1}(b+i s, b-i s ; b+c ;-z) \\
& =\Gamma(a+i s)(1+z)^{-b-i s}{ }_{2} F_{1}\left(b+i s, c+i s ; b+c ; \frac{z}{z+1}\right) \\
& =\Gamma(a+i s)(1+z)^{a-b}\left(1-\frac{z}{z+1}\right)^{a+i s}{ }_{2} F_{1}\left(b+i s, c+i s ; b+c ; \frac{z}{z+1}\right) .
\end{aligned}
$$

Используя производящую функцию для многочленов Хана (см. [30; с. 349]), мы получаем

$$
\Gamma(a+i s)(1+z)^{a-b} \sum_{k=0}^{\infty} \frac{S_{k}\left(x^{2} ; a, b, c\right)}{k !(b+c)_{k}}\left(\frac{z}{z+1}\right)^{k}
$$

а это равносильно нашему утверждению. 


\section{$\S 8$. Приложение $\Lambda$-функции и зазеркальных базисов. Симметрические пространства $\mathrm{U}(p, q) /(\mathrm{U}(p) \times \mathrm{U}(q))$}

8.1. Пространство $\mathrm{U}(p, q) /(\mathrm{U}(p) \times \mathrm{U}(q))$. Пусть $p \leqslant q$. Через $\mathrm{U}(p, q)$ мы обозначим псевдоунитарную группу порядка $(p, q)$, т.е. группу всех матриц $g=$ $\left(\begin{array}{ll}a & b \\ c & d\end{array}\right)$, удовлетворяющих условию

$$
\left(\begin{array}{ll}
a & b \\
c & d
\end{array}\right)\left(\begin{array}{cc}
1 & 0 \\
0 & -1
\end{array}\right)\left(\begin{array}{ll}
a & b \\
c & d
\end{array}\right)^{*}=\left(\begin{array}{cc}
1 & 0 \\
0 & -1
\end{array}\right)
$$

Через $\mathrm{B}_{p, q}$ мы обозначим множество всех матриц над $\mathbb{C}$ размера $p \times q$ с нормой $<1$. Группа $\mathrm{U}(p, q)$ действует на $\mathrm{B}_{p, q}$ дробно-линейньми преобразованиями

$$
z \mapsto z^{[g]}:=(a+z c)^{-1}(b+z d) .
$$

Стабилизатор $K$ точки 0 состоит из матрищ вида

$$
\left(\begin{array}{ll}
a & 0 \\
0 & d
\end{array}\right), \quad a \in \mathrm{U}(p), \quad d \in \mathrm{U}(q) .
$$

Пространство $\mathrm{B}_{p, q}$ является однородным симметрическим пространством

$$
\mathrm{B}_{p, q}=\mathrm{U}(p, q) /(\mathrm{U}(p) \times \mathrm{U}(q)) .
$$

Мера на пространстве $\mathrm{B}_{p, q}$, инвариантная относительно $\mathrm{U}(p, q)$, имеет плотность $\operatorname{det}\left(1-z z^{*}\right)^{-p-q}$ относительно меры Лебега. Группа $\mathrm{U}(p, q)$ действует в $L^{2}$ по инвариантной мере заменами переменной

$$
T(g) f(z)=f\left(z^{[g]}\right) .
$$

8.2. Пространства Березина $V_{\theta}^{\circ}\left(\mathrm{B}_{p, q}\right)$. Пространство Березина $V_{\theta}^{\circ}\left(\mathrm{B}_{p, q}\right)$ задается как пространство функций на $\mathrm{B}_{p, q}$, определенное ядром

$$
K(z, u)=\frac{\operatorname{det}\left(1-z z^{*}\right)^{\theta / 2} \operatorname{det}\left(1-u u^{*}\right)^{\theta / 2}}{\left|\operatorname{det}\left(1-z u^{*}\right)\right|^{\theta}} .
$$

Группа $\mathrm{U}(p, q)$ действует в $V_{\theta}^{\circ}\left(\mathrm{B}_{p, q}\right)$ по формуле (8.1). При достаточно больших $\theta$ представление $\mathrm{U}(p, q)$ в $V_{\theta}^{\circ}\left(\mathrm{B}_{p, q}\right)$ эквивалентно представлению в $L^{2}$ (см. [16], а также [18], [23]). Задача Эрстеда состоит в том, чтобы построить явно унитарный сплетающий оператор между этими представлениями.

8.3. $\Lambda$-функция пространства $\mathrm{U}(p, q) /(\mathrm{U}(p) \times \mathrm{U}(q))$. Обозначим через $\lambda_{1}(z)$, $\ldots, \lambda_{p}(z)$ сингулярные числа матрицы $z$, положим

$$
x_{j}(z)=\frac{\lambda_{j}^{2}(z)}{1-\lambda_{j}^{2}(z)} .
$$

Мы определяем $\Lambda$-функцию пространства $\mathrm{U}(p, q) /(\mathrm{U}(p) \times \mathrm{U}(q))$ по формуле

где

$$
\Lambda^{\theta}(z)=\operatorname{det}\left(\begin{array}{ccc}
\Xi_{0}\left(x_{1}\right) & \ldots & \Xi_{0}\left(x_{p}\right) \\
\Xi_{1}\left(x_{1}\right) & \ldots & \Xi_{1}\left(x_{p}\right) \\
\vdots & \ddots & \vdots \\
\Xi_{p-1}\left(x_{1}\right) & \ldots & \Xi_{p-1}\left(x_{p}\right)
\end{array}\right)
$$

$$
\Xi_{j}(x)=\Xi_{j}\left(x ; \theta-\frac{q+p-1}{2} ; \frac{q-p+1}{2} ; \frac{q-p+1}{2}\right)
$$

- первые $p$ элементов зазеркального базиса. 
Теорема 8.1 (см. также [41]). Для $g \in \mathrm{U}(p, q)$ через и мы обозначим образ точки $0 \in \mathrm{B}_{p, q}$ под действием $g$. Тогда

$$
\int_{\mathrm{B}_{p, q}} \Lambda^{\theta}(z) \overline{\Lambda^{\theta}\left(z^{[g]}\right)} \operatorname{det}\left(1-z z^{*}\right)^{-p-q} d z=\operatorname{det}\left(1-u u^{*}\right)^{\theta / 2}
$$

СлЕДСТВИЕ 8.2. Определим функиию $L(z, u)$ на $\mathrm{B}_{p, q} \times \mathrm{B}_{p, q}$ по формуле

$$
L(z, u)=\Lambda\left(z^{\left[g_{u}\right]}\right)
$$

әде $g_{u} \in \mathrm{U}(p, q)$ - любой элемент, переводящий точку $0 \in \mathrm{B}_{p, q}$ в точку и. Тогда оператор

$$
M f(z)=\int_{\mathrm{B}_{p, q}} L(z, u) f(u) \operatorname{det}\left(1-u u^{*}\right)^{-p-q} d u
$$

является унитарным $\mathrm{U}(p, q)$-сплетающим оператором

$$
L^{2}\left(\mathrm{~B}_{p, q}\right) \rightarrow V_{\theta}^{\circ}\left(\mathrm{B}_{p, q}\right)
$$

ЗАмЕчАниЕ. Определение ядра $L(z, u)$ можно переформулировать следующим образом. Оно задается определителем (8.2), где в качестве чисел $\lambda_{j}$ берутся сингулярные числа матрицы

$$
\left(1-z z^{*}\right)^{-1 / 2}\left(1-z u^{*}\right)\left(1-u u^{*}\right)^{-1 / 2}
$$

ЗАмЕчаниЕ. Аналогичная теорема верна для групп серий $\mathrm{O}(n, \mathbb{C})$ и $\operatorname{Sp}(2 n, \mathbb{C})$.

\section{Список литературы}

1. Weyl H. Über gewönliche lineare Differentialgleichungen mis singulären Stellen und ihre Eigenfunktionen (2 Note) // Nachr. Konig. Gess. Wissen. Göttingen. Math.-Phys. 1910. P. 442-467; Weyl H. // Gessamelte Abhandlungen. V. 1. Berlin: Springer-Verlag, 1968. P. 222-247.

2. Mehler F. G. Über eine mit den Kugel und Zylinderfunctionen verwandte Function und ihre Anwedung in der Theorie der Elektricitatsvertheilung // Math. Ann. 1881. V. 18. P. 161-194.

3. Тичмари Э. Ч. Разложения по собственньпм функциям, связанные с дифференциальными уравнениями второго порядка. Т. 1. М.: ИЛ, 1960.

4. Олевский M. Н. О представлении произвольной функции через интеграл с ядром, включающим гипергеометрическую функцию // Докл. АН СССР. 1949. Т. 69. № 1. С. 11-14.

5. Flensted-Jensen M., Koornwinder T. The convolution structure for Jacobi function expansions // Ark. Math. 1973. V. 11. P. 245-262.

6. Koornwinder T. H. A new proof of a Paley-Wiener theorem for Jacobi transform // Ark. Math. 1975. V. 13. P. 145-159.

7. Koornwinder T. H. Jacobi functions and analysis on noncompact symmetric spaces // Special functions: group theoretical aspects and applications / ed. R. Askey et al. Dodrecht: Reidel, 1984. P. 1-85.

8. Koornwinder T. H. Special orthogonal polynomial systems mapping to each other by Fourier-Jacobi transform // Lecture Notes in Math. 1985. V. 1171. P. 174-183. 
9. Yakubovich S. B., Luchko Yu.F. The hypergeometric approach to integral transforms and convolutions. Dordrecht: Kluwer Acad. Publ., 1994. (Math. Appl. (Dordrecht). V. 287.)

10. Самко С.Г., Килбас А. А., Маричев О. И. Интегралы и производные дробного порядка и некоторые их приложения. Минск: Наука и техника, 1987.

11. Прудников А.М., Брычков Ю.А., Маричев О.И. Интегралы и ряды. М.: Наука, 1981-1986.

12. Koelink E., Stockman J. V. Askey-Wilson transform scheme // Preprint, http://xxx.lanl. gov/math/9912140.

13. Heckman G. I., Opdam E. M. Root systems and hypergeometric functions. I // Compositio Math. 1987. V. 64. P. 329-352.

14. Heckman G. I. Root systems and hypergeometric functions. II // Compositio Math. 1987. V. 64. P. 353-373.

15. Opdam E. M. Root systems and hypergeometric functions. III, IV // Compositio Math. 1988. V. 67. P. 21-49; 191-209.

16. Березин $\Phi . A$. Связь между ко и контравариантными символами операторов на классических комплексных классических симметрических пространствах // Докл. АН СССР. 1978. T. 241. № 1. C. 15-17.

17. Неретин Ю.А., Ольшанский Г.И. Граничные значения голоморфных функций, особые унитарные представления групп $\mathrm{O}(p, q)$ и их пределы при $q \rightarrow 0 / /$ Записки научн. сем. ПОМИ. 1995. Т. 223. С. 9-91; // J. Math. Sci. New York. 1997. V. 87. №6. P. 3983-4035.

18. Olafsson G., Ørsted B. Generalizations of the Bargmann transform // Lie theory and its applications in physics. Proceedings of the international workshop, Clausthal, Germany, August 14-17, 1995 / ed. H.-D. Döbner et al. Singapore: World Scientific, 1996. P. 3-14.

19. van Dijk G., Hille $S$. C. Canonical representations related to hyperbolic spaces // J. Funct. Anal. 1997. V. 147. № 1. P. 109-139.

20. Hille S. C. Canonical representations // Ph.D. Thesis. Leiden University, 1999.

21. Неретин Ю. А. Матричные аналоги В-функции и формула Планшереля для керн-представлений Березина // Матем. сб. 2000. Т. 191. № 5. С. 67-100; // Preprint, http://xxx. lanl.gov/math.RT/9905045.

22. Неретин Ю. А. О разделении спектров в анализе ядер Березина // Функц. анализ и его прилож. 2000. Т. 34. № 3. C. 49-62; // Preprint, http://xxx.lanl.gov/math.RT/9906075.

23. Neretin Yu. A. Plancherel formula for Berezin deformation of $L^{2}$ on Riemannian symmetric space // Preprint, http://xxx.lanl.gov/abs/math/9911020 (to appear in J. Funct. Anal.).

24. Zhang Zhen Kai. Tensor products of weighted Bergman spaces and invariant Ha-plitz operators // Math. Scand. 1992. V. 71. P. 69-84.

25. Cherednik I. Harish-Chandra transform and difference operators // Preprint, http://xxx. lanl.gov.9706010.

26. Myller-Lebedeff W. Sur l'équation hypergéometrique // C.R. Acad. Sci. Paris. 1909. V. 149. P. 561-563.

27. Myller-Lebedeff $W$. Orthogonale hypergeometrische Functionen // Math. Ann. 1911. V. 70. P. 87-93.

28. Данфорд Н., Швари, Дж. Т. Линейные операторы. М.: Мир, 1966.

29. Бейтмен Г., Эрдейи А. Высшие трансцендентные функции. Т. 1-3. М.: Наука, 1965, 1966,1967

30. Andrews G. E., Askey R., Roy R. Special functions. Cambridge: Cambridge Univ. Press, 1999.

31. Vilenkin N. Ya., Klimyk A. U. Representations of Lie groups and special functions. V. 1. Dordrecht: Kluwer Acad. Publ., 1991. (Math. Appl. (Soviet Ser.). V. 72.)

32. Градитейн И. С., Рыљик И. М. Таблицы интегралов, сумм, рядов и произведений. М.: Физматгиз, 1963.

33. Gross K.I., Richards D. S. P. Total positivity, spherical series, and hypergeometric functions of matrix argument // J. Approx. Theory. 1989. V. 59. P. 224-246.

34. Odzijewicz A. Quantum algebras and $q$-special functions related to coherent states maps of the disk // Comm. Math. Phys. 1998. V. 192. P. 183-215. 
35. Slater L. J. Generalized hypergeometric functions. Cambridge: Cambridge Univ. Press, 1966.

36. Маричев О. И. Методы вычисления интегралов от специальных функций. Минск: Наука и техника, 1978.

37. Volterra $V$. Teoria delle potenze dei logaritmi e delle funzioni di decomposizione // Mem. Accad. Lincei. Ser. 5. 1916. V. 11. № 4. P. 167-250.

38. Humbert P., Poli L. Sur certaines transcendentes liees au calcul symbolique // Bull. Sci. Math. (2). 1944. V. 68. P. 204-214.

39. Colombo $S$. Sur quelques nouvelles correspondances symboliques // Bull. Sci. Math. (2). 1943. V. 67. P. 104-107.

40. McLachlan N. W., Humbert P., Poli L. Supplement au formulaire pour le calcul symbolique. Paris: Gauthier-Villars, 1950. (Mem. Sci. Math. V. 113.)

41. Neretin Yu.A. Matrix balls, radial analysis of Berezin kernels, and hypergeometric determinants // Preprint, http://xxx.lanl.gov/abs/math/0012220 (to appear in Moscow Math. J.).

Институт теоретической и экспериментальной физики,

Поступила в редакцию 